Greater Everglades Priority Ecosystems Science

\title{
User's Manual for the Automated Data Assurance and Management Application Developed for Quality Control of Everglades Depth Estimation Network Water-Level Data
}

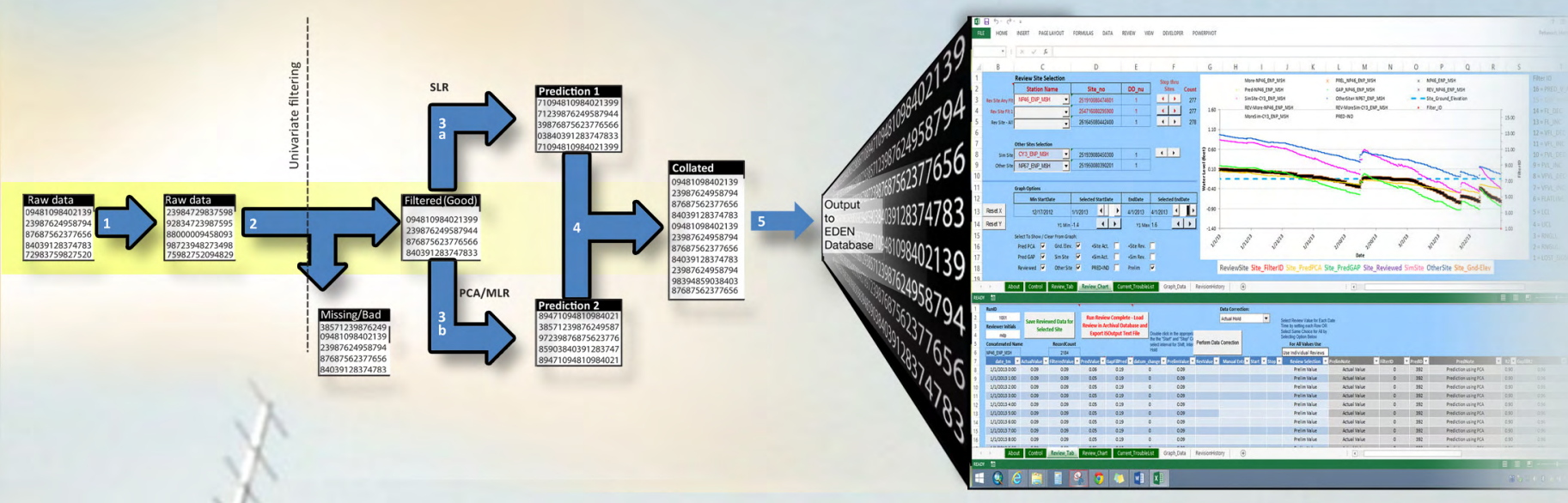

Open-File Report 2016-1116

U.S. Department of the Interior U.S. Geological Survey 


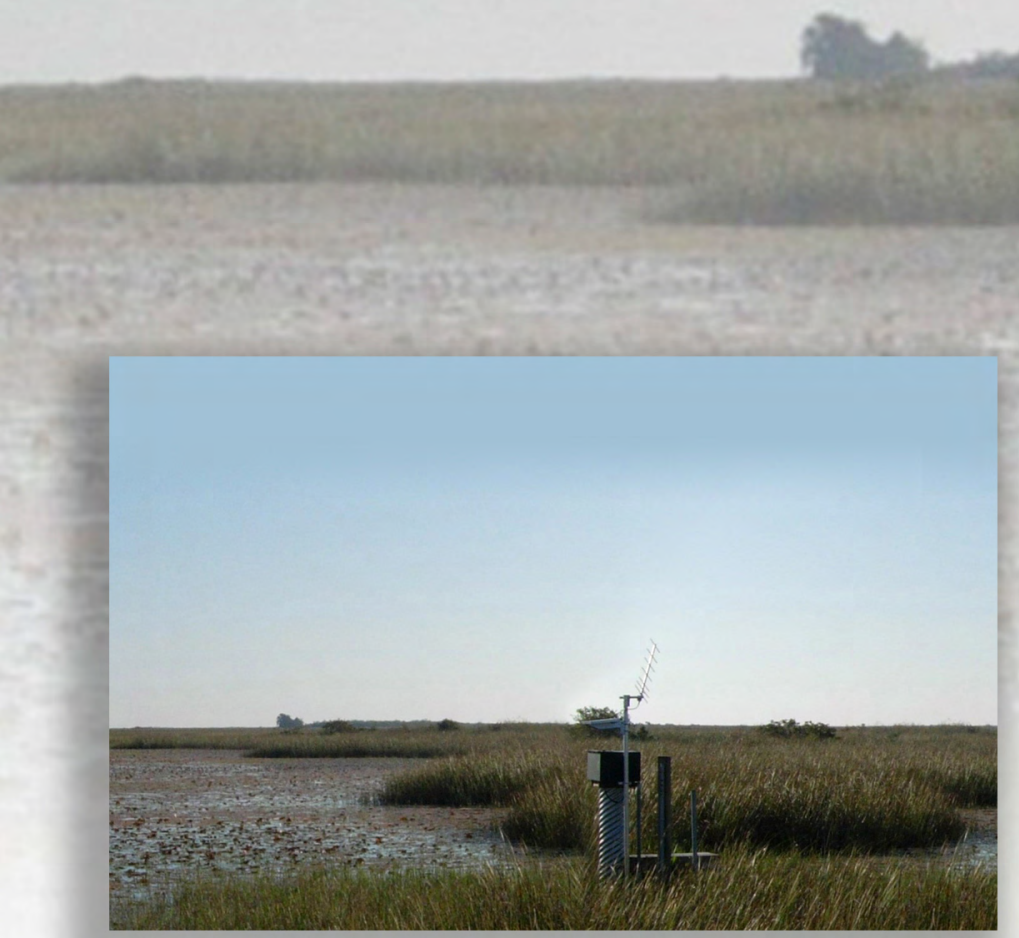

Cover. Gaging station W11 in Water Conservation Area 3A of the Florida Everglades, Broward County, Florida. Photograph by Christa Zweig, South Florida Water Management District, West Palm Beach, Florida. 


\section{User's Manual for the Automated Data Assurance and Management Application Developed for Quality Control of Everglades Depth Estimation Network Water-Level Data}

By Matthew D. Petkewich, Ruby C. Daamen, Edwin A. Roehl, and Paul A. Conrads

Greater Everglades Priority Ecosystems Science

Open-File Report 2016-1116 


\title{
U.S. Department of the Interior SALLY JEWELL, Secretary
}

\section{U.S. Geological Survey Suzette M. Kimball, Director}

\author{
U.S. Geological Survey, Reston, Virginia: 2016
}

For more information on the USGS - the Federal source for science about the Earth, its natural and living resources, natural hazards, and the environment—visit http://www.usgs.gov or call 1-888-ASK-USGS.

For an overview of USGS information products, including maps, imagery, and publications, visit http://store.usgs.gov.

Any use of trade, firm, or product names is for descriptive purposes only and does not imply endorsement by the U.S. Government.

Although this information product, for the most part, is in the public domain, it also may contain copyrighted materials as noted in the text. Permission to reproduce copyrighted items must be secured from the copyright owner.

Suggested citation:

Petkewich, M.D., Daamen, R.C., Roehl, E.A., and Conrads, P.A., 2016, User's manual for the Automated Data Assurance and Management application developed for quality control of Everglades Depth Estimation Network waterlevel data: U.S. Geological Survey Open-File Report 2016-1116, 28 p., http://dx.doi.org/10.3133/ofr20161116.

ISSN 2331-1258 (online) 


\section{Contents}

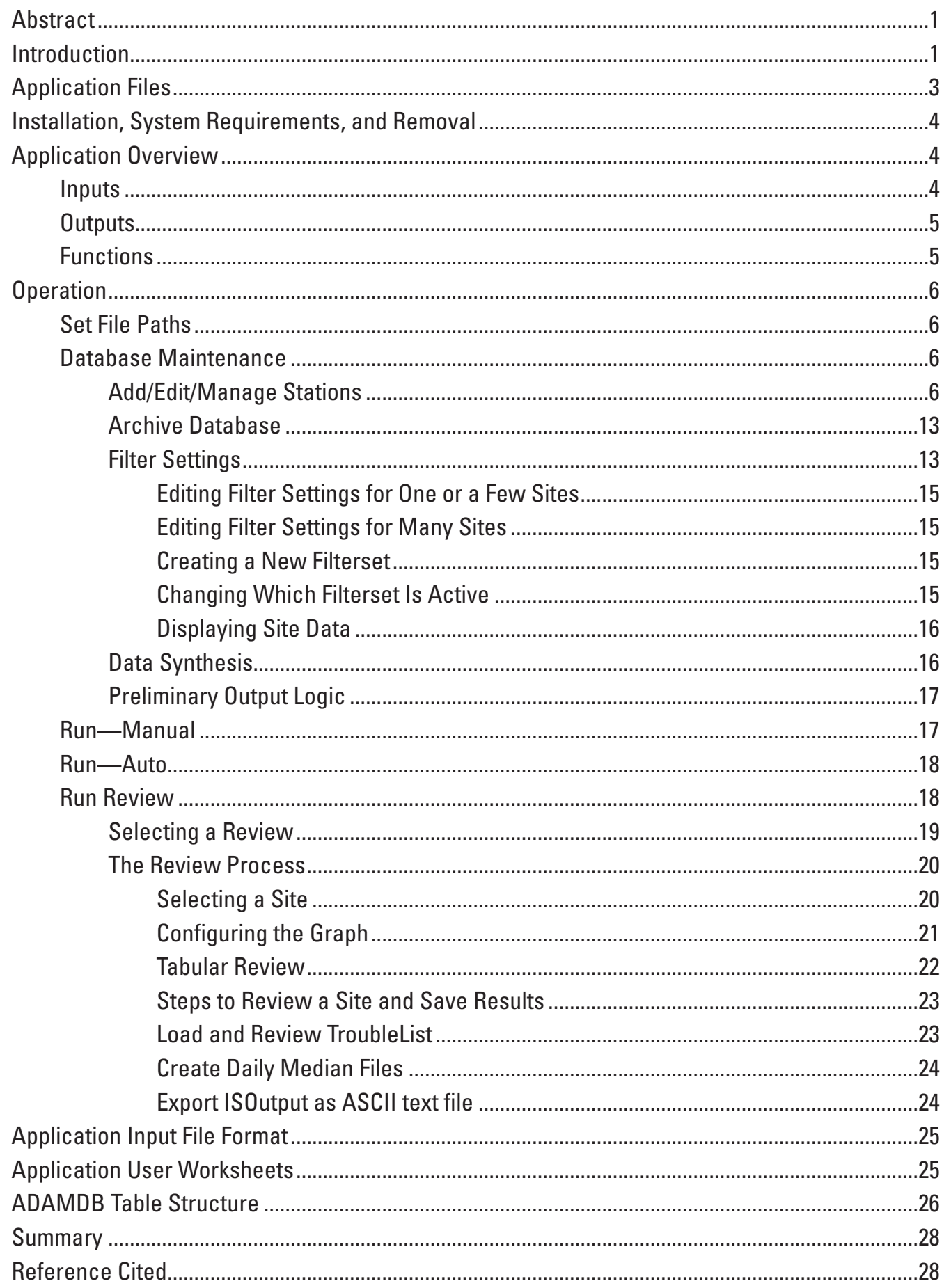




\section{Figures}

1. Map showing location of the water-level stations within the Everglades Depth Estimation Network

2. Schematic diagram showing flow of Everglades Depth Estimation Network data from agency databases to the Web.

3. Screen capture showing section of Contro/ worksheet used for file path selection .......6

4. Screen capture showing how selecting "Maintain ADAM Stations" from the Control worksheet will take the user to a split window showing the ADAMSiteList and UserSiteList worksheets.

5. Screen captures showing partial views of ADAMSiteList and UserSiteList worksheets

6. Screen capture showing form used to add stations to ADAMDB .....................................9

7. Screen capture showing partial view of Gapfill worksheet..........................................10

8. Screen capture of the "Add Gapfill Sites" form used to add Gapfill sites for a site .......11

9. Screen capture showing example of editing Gapfill equations for a site ........................12

10. Screen captures showing examples of archive prompt displayed in "Database Maintenance" section of the Contro/worksheet when file size exceeds 800 megabytes and userform displayed after selecting "Archive" command in initial prompt.

11. Screen capture showing upper left portion of Filter_Adjustments worksheet................14

12. Screen capture showing bottom portion of Filter_Adjustments worksheet ....................14

13. Screen capture showing graphical display of available site data from the

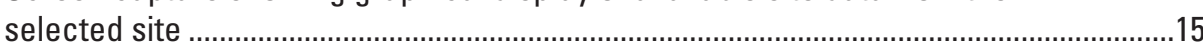

14. Screen capture showing the Fill Setup area of the Contro/ worksheet ...........................16

15. Screen capture of PrelimLogic worksheet showing logic used by ADAM to set a preliminary value for each station ..............................................................................17

16. Screen capture showing Contro/worksheet "Run Setup and Execution" controls .......18

17. Screen capture showing Contro/worksheet—run progress and tabular run history....18

18. Screen capture showing the section of the Control worksheet used to set file paths.

19. Screen capture showing the section of the Contro/ worksheet used to select run for review

20. Screen capture showing the section of the Contro/worksheet used to manually enter a run for review

21. Screen capture showing Review_Chartworksheet—site selection and graph setup.

22. Screen capture showing a sample graph display generated using the Review_Chartworksheet.

23. Screen capture showing Review_Tab worksheet—partial view..

24. Screen capture showing display of prediction details accessed from the Review_Tab worksheet.

25. Screen capture showing Current_TroubleList worksheet.

26. Screen capture showing Contro/worksheet commands used to generate daily median files and export data. 


\section{Tables}

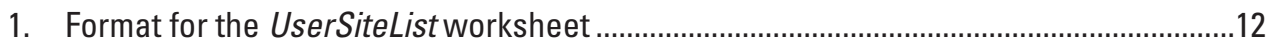

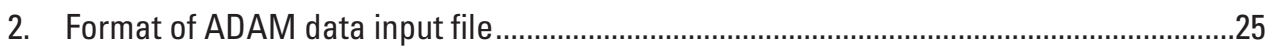

3. ADAM worksheets that are accessed for setting up or completing an ADAM run........25

4. ADAM worksheets that are accessed for completing an ADAM review........................25

5. ADAM_Setup.mdb tables and queries required by ADAM ............................................26

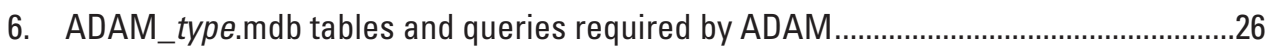

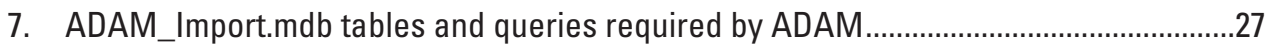

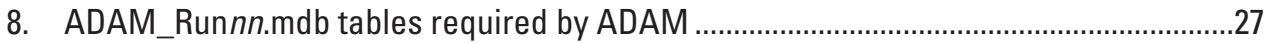

9. ADAM_Query.mdb tables and queries required by ADAM ...........................................28

\section{Conversion Factors}

International System of Units to U.S. customary units

\begin{tabular}{lcc}
\hline Multiply & By & To obtain \\
\hline meter $(\mathrm{m})$ & Length & \\
\hline
\end{tabular}




\section{Abbreviations}

$\begin{array}{ll}\text { ASCII } & \text { American Standard Code for Information Exchange } \\ \text { ADAM } & \text { Automated Data Assurance and Management } \\ \text { CERP } & \text { Comprehensive Everglades Restoration Plan } \\ \text { EDEN } & \text { Everglades Depth Estimation Network } \\ \text { MB } & \text { megabyte } \\ \text { MLR } & \text { multivariate linear regression } \\ \text { NWIS } & \text { National Water Information System } \\ \text { PCA } & \text { principal component analysis } \\ \text { SLR } & \text { simple linear regression } \\ \text { SPC } & \text { statistical process control } \\ \text { USGS } & \text { U.S. Geological Survey } \\ \text { VBA } & \text { visual basic for applications }\end{array}$




\title{
User's Manual for the Automated Data Assurance and Management Application Developed for Quality Control of Everglades Depth Estimation Network Water-Level Data
}

\author{
By Matthew D. Petkewich, ${ }^{1}$ Ruby C. Daamen, ${ }^{2}$ Edwin A. Roehl, ${ }^{2}$ and Paul A. Conrads ${ }^{1}$
}

\begin{abstract}
The generation of Everglades Depth Estimation Network (EDEN) daily water-level and water-depth maps is dependent on high quality real-time data from over 240 water-level stations. To increase the accuracy of the daily water-surface maps, the Automated Data Assurance and Management (ADAM) tool was created by the U.S. Geological Survey as part of Greater Everglades Priority Ecosystems Science. The ADAM tool is used to provide accurate quality-assurance review of the real-time data from the EDEN network and allows estimation or replacement of missing or erroneous data. This user's manual describes how to install and operate the ADAM software. File structure and operation of the ADAM software is explained using examples.
\end{abstract}

\section{Introduction}

The Everglades Depth Estimation Network (EDEN) is an integrated network of 247 real-time water-level gaging stations (fig. 1), a ground-elevation model, and a water-surface elevation model designed to provide scientists, engineers, and waterresource managers with current (1991-2016) water-level and water-depth information for the entire freshwater portion of the Everglades. The EDEN is presented on a grid consisting of more than fifty-thousand 400 meter $(\mathrm{m}) \mathrm{x} 400 \mathrm{~m}$ grid cells and offers a consistent and documented dataset that can be used by scientists and water-resource managers to (1) guide large-scale field operations, (2) integrate hydrologic and ecological data and analysis, and (3) support biological and ecological assessments that measure ecosystem responses to the Comprehensive Everglades Restoration Plan (CERP). In addition, EDEN, with the integration of real-time data and models, provides opportunities for real-time evaluation of water-level conditions and water-resource management operation. The EDEN database is a 26-year dataset of baseline conditions (1991-2016) prior to the full implementation of CERP and offers investigators a single repository for historic daily and hourly water-level data.

The generation of daily EDEN water-level surfaces is dependent on high quality, real-time data because missing or erroneous data can compromise the quality of the modeled water-surface maps. Data for the network may be missing because of instrumentation failure or data telemetry transmission problems. When data from a particular station are missing, the watersurface model does not use that station for generating the water-surface map for that day. The quality of the water-surface maps therefore can be diminished, depending on the number of stations with missing data and the locations of those stations.

In order to provide daily modeled water surfaces on a near real-time basis, EDEN needed an automated process to identify errors in water-level data and to provide estimates for the missing or erroneous data. To increase the accuracy of the daily water-surface maps, the Automated Data Assurance and Management (ADAM) tool was created by the U.S. Geological Survey (USGS) as part of Greater Everglades Priority Ecosystems Science to facilitate an accurate quality-assurance review of the realtime data from the EDEN network and allow estimation or replacement of missing or erroneous data. Incorporation of ADAM in the review process of all EDEN datasets has improved the consistency and utility of the EDEN data.

This user manual describes how to install and operate the ADAM application, which is a Microsoft Excel and Access database tool that uses a variety of signal processing and intelligent software technologies in order to automate the validation and correction of the data on a near real-time basis. As shown in figure 2, ADAM is located between the individual EDEN partner agency databases (DBHYDRO, DataForEver, and the National Water Information System [NWIS]) and the internal EDEN database, so that by the time the agency data are loaded into the EDEN database, all missing and erroneous data have been addressed. Petkewich and others (2016) documents the development of the ADAM software for EDEN and includes examples of the methods used to identify errors in real-time water-level data and provide estimates for that data.

${ }^{1}$ U.S. Geological Survey.

${ }^{2}$ Advanced Data Mining International. 


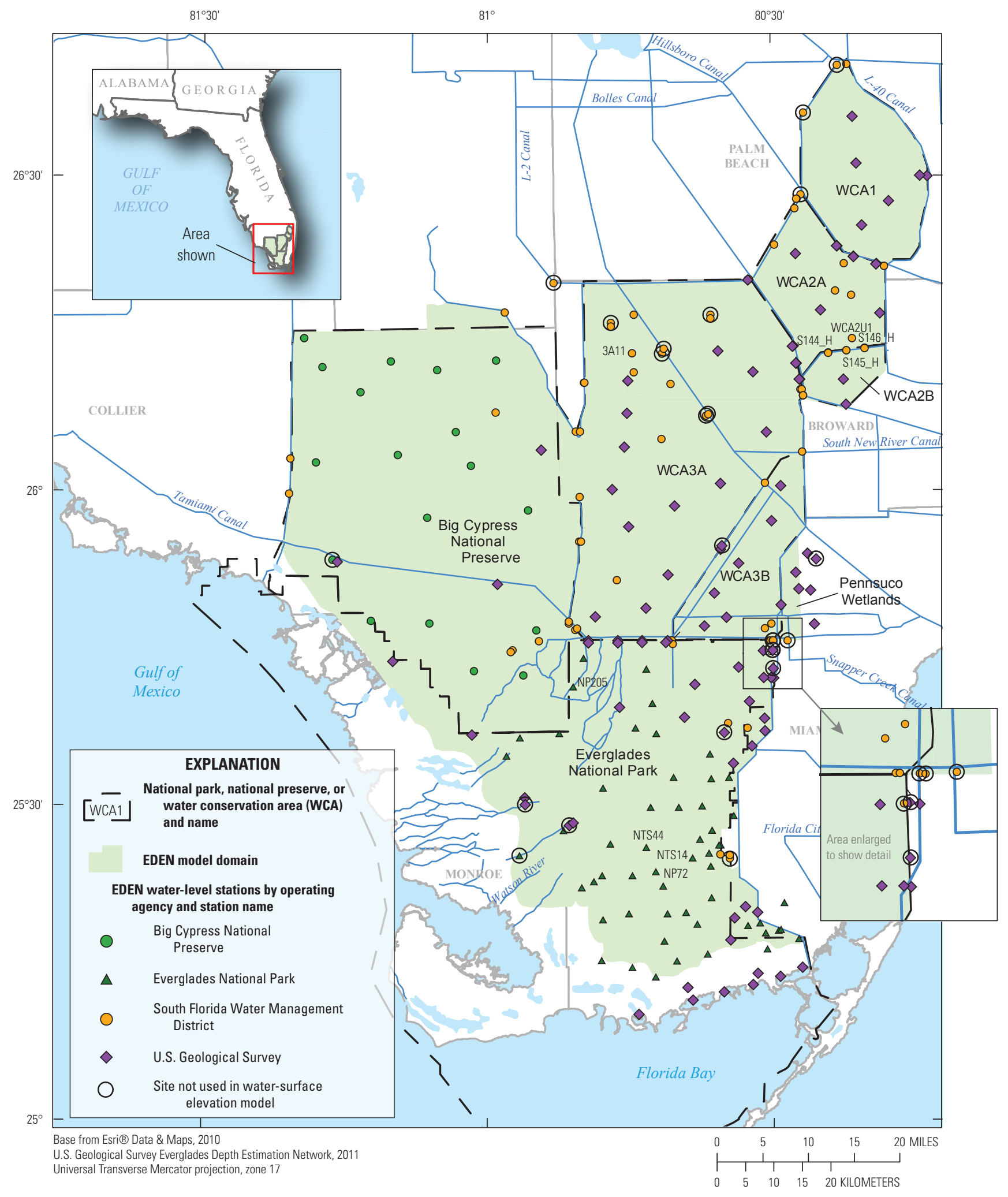

Figure 1. Location of the water-level stations within the Everglades Depth Estimation Network (EDEN). 


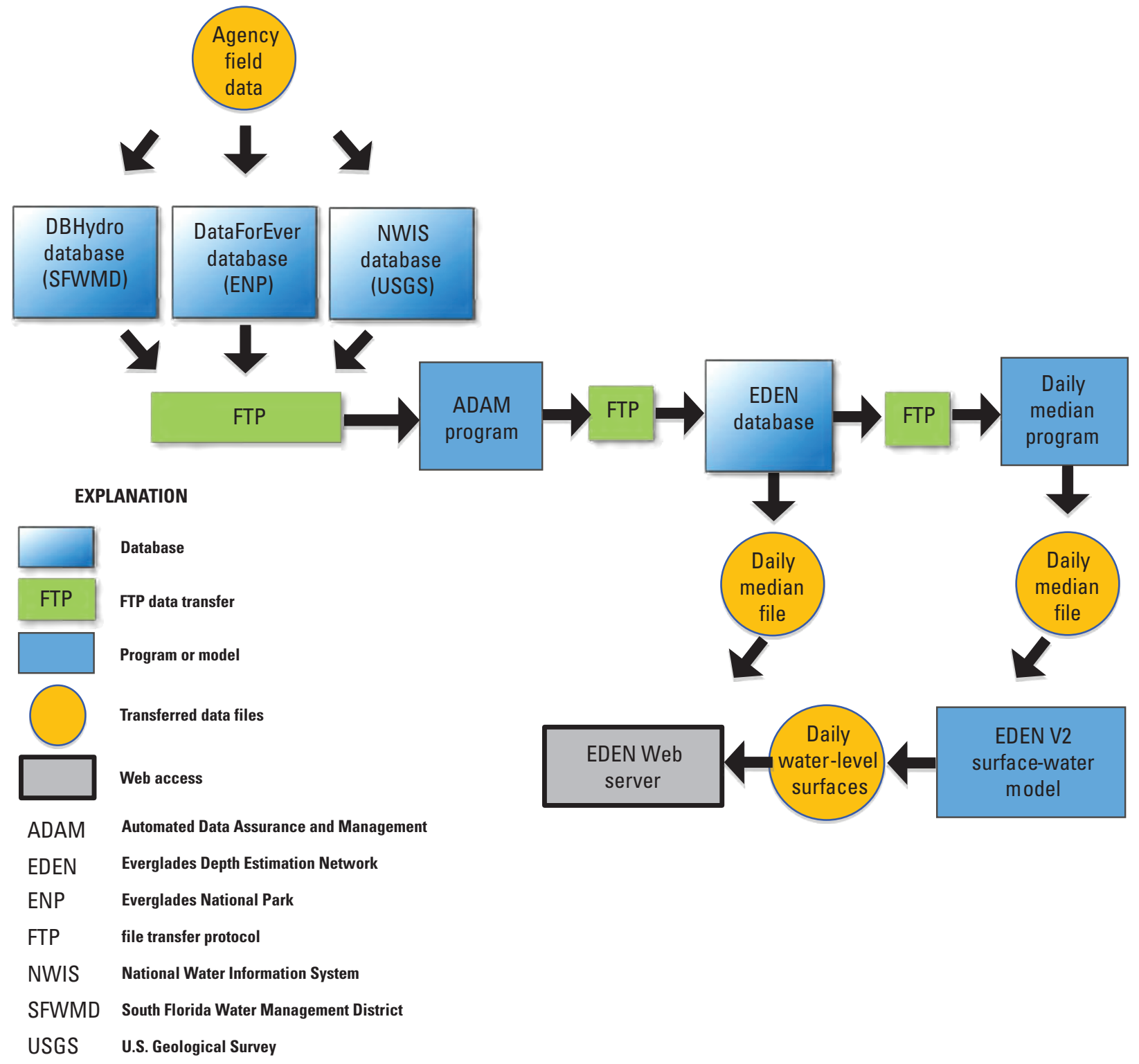

Figure 2. Flow of Everglades Depth Estimation Network data from agency databases to the Web.

\section{Application Files}

ADAM consists of the following files located in the ADAM directory:

1. Microsoft Excel application

- ADAM.xlsm/ADAM_Auto.xlsm (ADAM)

- ADAM_Review.xlsm (ADAM_REVIEW)

2. Microsoft Access database files (ADAMDB):

- ADAM_daily.mdb

- ADAM qtly.mdb

- ADAM_annual.mdb 
- ADAM_Setup.mdb (ADAM_SETUP)

- ADAM_Import.mdb

- ADAM_Query.mdb

3. ADAM_UserManual.doc-Microsoft Word

4. Subdirectories

A. inputfiles

- Default location for ADAM input files

- Schema.ini

- Schema_reference.ini

B. outputfiles

- Default location for ADAM output files

5. Archives

\section{Installation, System Requirements, and Removal}

The ADAM application files are available for download at http://dx.doi.org/10.3133/ofr20161116. To install the application, the user copies the ADAM folder to their computer hard drive. The user may rename the ADAM folder. The macro security level of Excel must be set to either medium or low by selecting Tools $>$ Macro $>$ Security. ADAM uses visual basic for applications (VBA) macros for a variety of purposes and must be able to execute them to operate correctly. ADAM was developed by using Excel for Office 2010 and Access for Office 2010 and may use features not available in earlier versions of Excel and Access. Best results are obtained by running ADAM_REVIEW on a computer with a large monitor. The ADAM application can be removed by deleting the folder containing the application and its contents.

\section{Application Overview}

All code (VBA macros) and user controls to run ADAM and ADAM_REVIEW are contained in each respective application. All data used and generated by ADAM are stored in ADAMDB. The following formats are used herein for referring to Access data and Excel commands:

- References to Access database files, tables, and field names have the following format: [Access filename].tablename. fieldname.

- References to Excel controls are made using the control caption between quotation marks.

A listing of ADAM user worksheets and a listing of ADAMDB files, tables, and fields are provided later in this user manual.

\section{Inputs}

An ASCII file (called "data_uv.txt" for daily automated runs) constructed using data extracted from NWIS and data from partner agency databases, is processed to NWIS output data format. The required file format is specified in the Application Input File Format section. The ASCII file for quarterly manual runs can be named "data_uv.txt" or another name if desired. The name will be specified during manual ADAM runs. 


\section{Outputs}

- ADAM_Runnn.mdb ( $n n=$ numeric RunID) —Access file containing the output for each ADAM run. These files are created from an ADAM run and used for manual data review using the ADAM_REVIEW workbook.

- Output text file - Output data file (ASCII text file) containing measured and predicted data for a specific ADAM run.

- Optional daily median files (ASCII text file) - Output data files (ASCII text files) that can be used to support the watersurface model.

\section{Functions}

1. Import data for analysis - Imports data from the input text file (called "data_uv.txt" for the daily runs) into ADAMDB. ADAM will read in data at hourly or 15-minute time steps for daily runs and only hourly data for quarterly or annual runs. Any data not read by ADAM are temporarily stored in the table [ADAM_Import.mdb].tbl_unreaddata_type (annual, quarterly, or daily). This table will be overwritten on any subsequent run of the same type.

2. Analyze data by sequencing two algorithms - statistical process control and regression models (explained in the main body of the report)

A. Statistical process control (SPC) series of 14 univariate filters. Filters can be set within ADAM.

B. Synthesize data using two different empirical models:

I. Multivariate linear regression (MLR) models—generated "on-the-fly" using principal component analysis (PCA). The MLR is configurable within the ADAM.xlsm Control worksheet. Note that the MLR models are labeled as "PCA" model on many of the screens in the ADAM application.

II. Simple linear regression (SLR) models - referred to as "Gapfill" herein. These models were developed for each EDEN station using data collected from March 1, 2006, through September 30, 2011, and stored in the ADAM database (ADAM_Setup.mdb).

\section{Facilitate user review of data-}

A. Users can view analysis output in tabular and graphical format.

B. Reviewed data are saved to ADAMDB.

4. Create output database file - Generate unique output database for each run (ADAM_Runnn.mdb, where $n n=$ unique runid).

5. Maintain history of analysis - Date of analysis and reviewer initials are stored in the database, along with review type (daily, quarterly, annual) and archived database name, if archived.

6. Generate daily median files_-Optional export function, from the ADAM_Review.xlsm Control worksheet, that supports the water-surface model. 


\section{Operation}

The following operations can be performed with the ADAM Excel application. The worksheet(s) used for each operation are described and screenshots provided.

\section{Set File Paths}

Workbook/Worksheet-ADAM.xlsm/Control

Description - Data paths for files used by ADAM must be set only if different than the default displayed (fig. 3). Figure 3 shows the section of the Control worksheet used to set the data paths. The user selects the appropriate command button to make the selection. The paths to set are as follows:

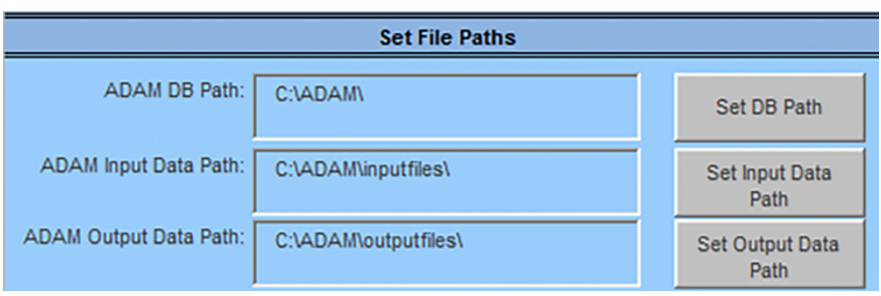

Figure 3. Contro/ worksheet used for file path selection. If the default paths shown are used (for example, C:IADAMI), no change is required.

- ADAM DB Path—By default, the directory in which ADAM.xlsm resides. All .mdb files must be located in the selected directory.

- ADAM Input Data Path - The directory where the input .txt files will be located. The files schema.ini and schema_reference.ini, which are included in the ADAM file set, also must be stored in the input data directory.

- ADAM Output Data Path-The directory where all ADAM output will be saved.

\section{Database Maintenance}

Maintenance of stations, filter settings, SLR equations, database synthesis, archival, and output logic can be configured with the ADAM Excel application. The worksheet(s) used for each operation are described and screenshots provided.

\section{Add/Edit/Manage Stations}

(The terms "gage," "site," and "station" are used interchangeably throughout the user manual.)

Workbook/Worksheets—ADAM.xlsm/Control, ADAMSiteList, UserSiteList, Gapfill

Description — All stations to be analyzed by ADAM are stored in [ADAM_Setup].tbl_StationList. This table can be modified by using the "Database Maintenance" section of the Control worksheet (fig. 4). Selecting this option will move control to two worksheets, ADAMSiteList and UserSiteList (fig. 5).

\section{To edit a station}

A. Make desired changes in appropriate column of the ADAMSiteList worksheet (fig. $5 A$ ). Required field formats can be displayed by rolling over the column header. Column headers with a bold lettering indicate required fields.

B. Cancel any unwanted edits prior to saving by selecting the "Cancel Edits AND Restore ADAM Station List" command.

C. Select the "Save Edits to ADAM Station List" button once all changes have been made.

D. Restoring a backup of tbl_StationList. Restoring and (or) deletion of tbl_StationList backups is completed within the ADAM_Setup.mdb database file. Prior to saving the edits, a backup copy of the current StationList will be saved to ADAM_Setup.mdb. The backup copies can be used to restore the current station list to an earlier setting. Users can restore backup copies by removing or renaming the current tbl_StationList file and then renaming the backup copy "tbl_StationList". Backup copies can be deleted at any time. 
Note: Information in the site_no and dd_nu columns cannot be changed in the UserSiteList and ADAMSiteList worksheets, respectively. In the ADAMSiteList worksheet, the user can change a usesite cell to FALSE if a station is no longer used.

\section{To add a station}

A. Enter data into the appropriate columns on the UserSiteList worksheet (fig. $5 B$, table 1).

B. Double-click on the station_name cell to open up a userform named "Add Site To ADAM Database" (fig. 6). Enter all required data as well as any other desired data.

C. Select the "ADD TO ADAM DB StationList" command to add the station to the database table once all required fields have been entered. The added station will display in the top row of the station list in the ADAMSiteList worksheet (fig. $5 A$ ).

3. To manage Gapfill equations (SLR), select the "Manage GAPFILL Equations" command on ADAMSiteList worksheet (fig. $5 A$ ) to go to the Gapfill worksheet (fig. 7) and view Gapfill equations. Users can do the following in the Gapfill worksheet:

A. Verify Gapfill sites. Site verification will list the following:

- Discontinued Gapfill stations that are assigned to active stations. Users can double-click on the station in the SOURCE SITE_NO column to change the usesite (ADAMSiteList worksheet, fig. 5A) field to TRUE or delete the Gapfill equation by double-clicking on the cell to the left (DEL column, Gapfill worksheet, fig. 7) of the Gapfill equation to delete.

- Active sites that do not have any Gapfill site assignments. Gapfill sites are not required; however, sites can be added by double-clicking the station in the SOURCE SITE_NO cell (fig. 7). Complete the displayed userform (fig. 8) to add the Gapfill equations.

\section{B. Add/remove Gapfill equations for active sites}

- To add additional Gapfill equations for a given site, double-click on the appropriate SITE_NO cell in the tabular list of equations (fig. 7). Enter the required information on the displayed userform (fig. 9) to add new equations.

- To remove Gapfill equations, double-click directly beneath "DEL" in the appropriate station row for the equation to be deleted (fig. 7). Alternatively, double click on the SITE_NO in the tabular list of equations and select the "Delete" command (fig. 9). Close the userform by clicking on the " $x$ " in the upper right corner of the form to cancel the deletion.

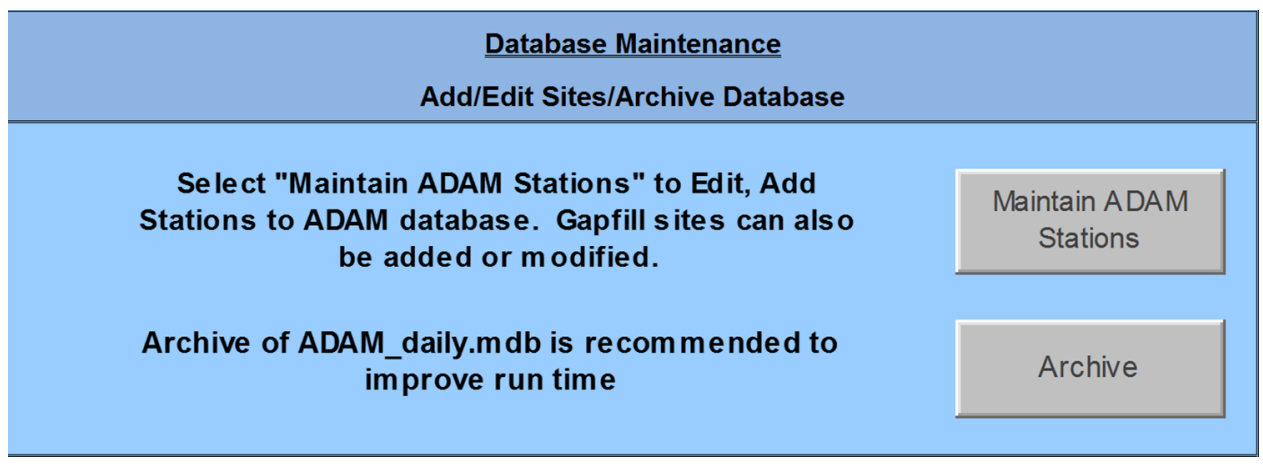

Figure 4. Selecting "Maintain ADAM Stations" from the Contro/ worksheet will take the user to a split window showing the ADAMSiteList and UserSiteList worksheets. 
$\boldsymbol{A}$

\begin{tabular}{|c|c|c|c|c|c|}
\hline ADAM Station List & site_no & dd_nu & station_name & usesite & concatenatedName \\
\hline \multirow{3}{*}{$\begin{array}{l}\text { Directions: } \\
\text { ADD Sites from the } \\
\text { UserSiteList worksheet }\end{array}$} & 02286700 & 2 & S8_DN & TRUE & S8T_WCA3_CSTR \\
\hline & 02287497 & 30 & NWWF & TRUE & NWWF_PENN_CNL \\
\hline & 02289019 & 16 & S12B_DN & TRUE & S12BT_ENP_MSTR \\
\hline \multirow{6}{*}{$\begin{array}{l}\text { Edit Sites at right. Site_no } \\
\text { and DD_nu cannot be } \\
\text { updated. Set UseSite to } \\
\text { False, if no longer used. }\end{array}$} & 02289019 & 24 & S12B_UP & TRUE & S12BH_WCA3_CSTR \\
\hline & 02289041 & 15 & S12C_DN & TRUE & S12CT_ENP_MSTR \\
\hline & 02289041 & 3 & S12C_UP & TRUE & S12CH_WCA3_CSTR \\
\hline & 02289050 & 1 & $\mathrm{~S} 333+\mathrm{H}$ & TRUE & S333H_WCA3_CSTR \\
\hline & 02289050 & 2 & $\mathrm{~S} 333+\mathrm{T}$ & TRUE & S333T_WCA3_CSTR \\
\hline & 022907647 & 1 & L31N_1 & TRUE & L31N1_ENP_CNL \\
\hline \multirow[t]{2}{*}{ Save Edits to ADAM Station List } & 02290765 & 1 & L31N_3 & TRUE & L31N3_ENP_CNL \\
\hline & 02290766 & 1 & L31N_4 & TRUE & L31N4_ENP_CNL \\
\hline \multirow{2}{*}{$\begin{array}{c}\text { Cancel Edits AND Restore ADAM } \\
\text { Station List }\end{array}$} & 02290767 & 1 & L31N_5 & TRUE & L31N5_ENP_CNL \\
\hline & 02290768 & 1 & L31N_7 & TRUE & L31N7_ENP_CNL \\
\hline \multirow[t]{2}{*}{ Manage GAPFILL Equations } & 02290769 & 7 & S18C_DN & TRUE & S18CT_ENP_CSTR \\
\hline & 02290810 & 1 & P37 & TRUE & P37_ENP_MSH \\
\hline Manage Station Filter Settings & 02290811 & 1 & NP206 & TRUE & NP206_ENP_MSH \\
\hline \multirow{2}{*}{ Return to CONIROL Worksheet } & 02290815 & 1 & P33 & TRUE & P33_ENP_MSH \\
\hline & 02290820 & 1 & P38 & TRUE & P38_ENP_MSH \\
\hline
\end{tabular}

B

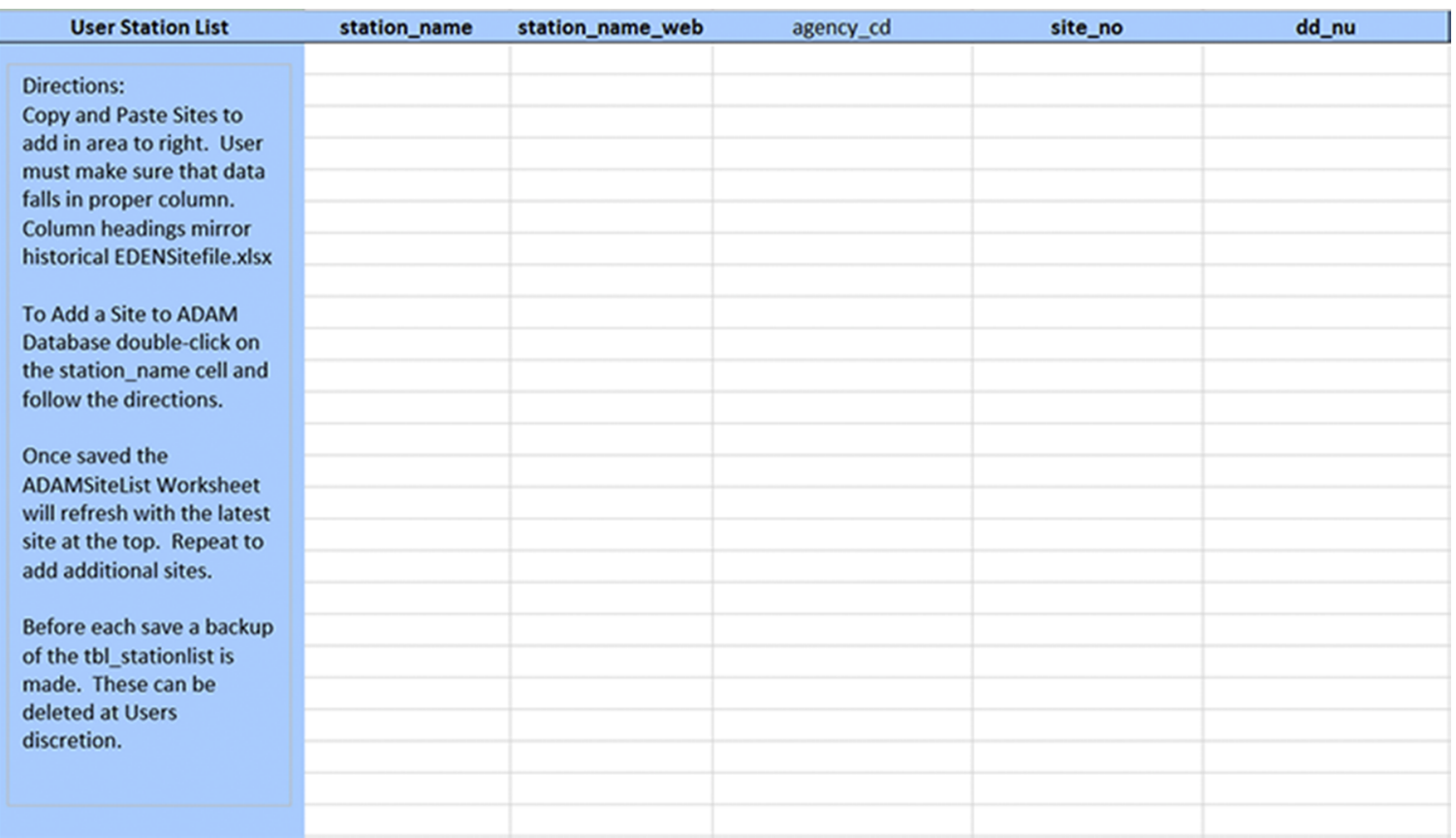

Figure 5. Partial views of, $A, A D A M S i t e L i s t$, and $B$, UserSite List worksheets. ADAMSiteList displays all records contained in [ADAM_SETUP].tbl_StationList. Stations can be edited from ADAMSiteList. New stations are added from UserSiteList. 


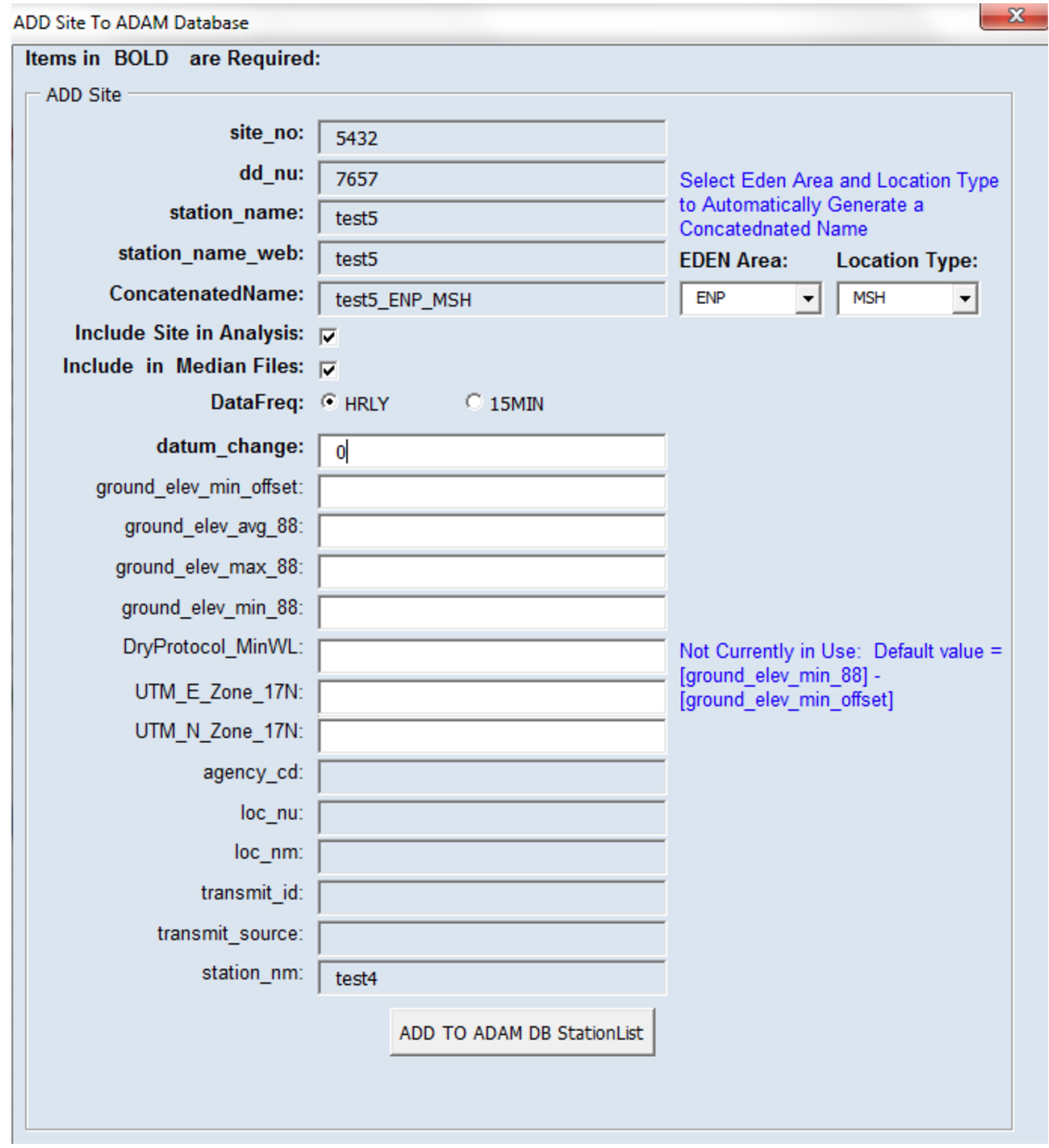

Figure 6. Form used to add stations to ADAMDB. Fields in bold type are required. The "ConcatenatedName" field is generated automatically after the user enters the "EDEN Area" and "Location Type" information using the provided drop-down menus. Data frequency is selected using the provided option buttons. 


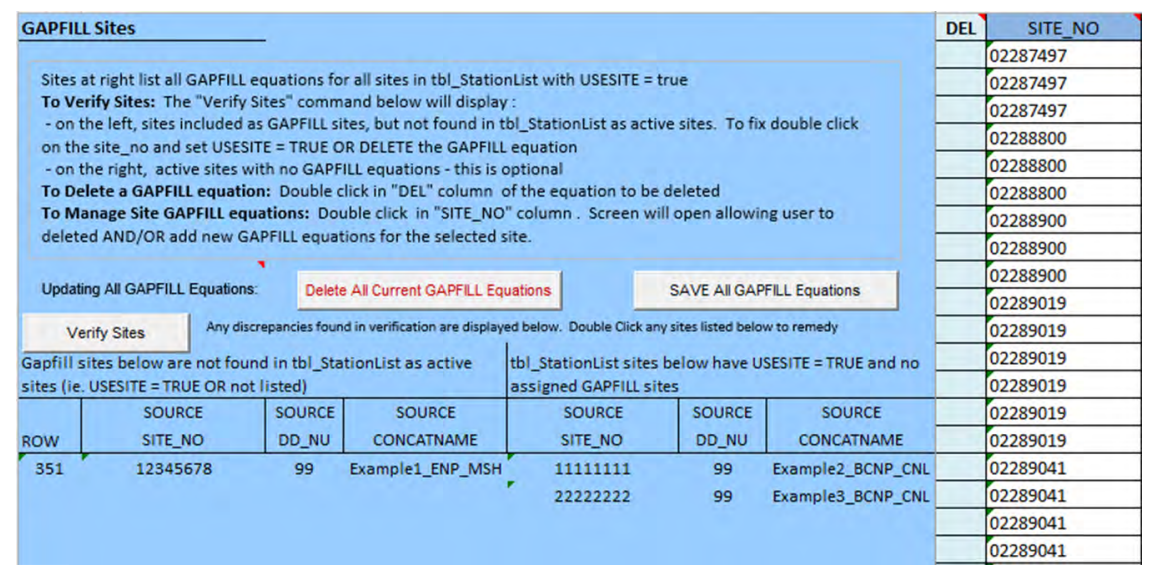

\begin{tabular}{|c|c|c|c|c|c|c|c|c|c|c|c|}
\hline DEL & SITE_NO & DD_NU & Concatenated Name & PrediD & PredstatusID & SimSite1_SITENO & SimSite1_DDNU & SimSite1_ConcatName & Const & Coef1 & pearson_corr_coef \\
\hline & 02287497 & 30 & NWWF_PENN_CNL & 259 & 5 & 255035080255402 & 1 & G-3761_PENN_MSH & 0.60 & 1.08 & 0.95 \\
\hline & 02287497 & 30 & NWWF_PENN_CNL & 258 & 5 & 255358080260901 & 1 & G-3567_PENN_MSH & -1.71 & 1.41 & 0.95 \\
\hline & 02287497 & 30 & NWWF_PENN_CNL & 257 & 5 & 255208080274001 & 1 & G-975_ENP_MSH & -1.81 & 1.26 & 0.94 \\
\hline & 02288800 & 4 & Monroe_BCNP_CNL & 3 & 5 & 255325081161300 & 1 & BCA8_BCNP_MSH & 2.33 & 1.24 & 0.98 \\
\hline & 02288800 & 4 & Monroe_BCNP_CNL & 2 & 5 & 254734081120900 & & BCA19_BCNP_MSH & 2.94 & 1.48 & 0.90 \\
\hline & 02288800 & 4 & Monroe_BCNP_CNL & 1 & 5 & 254721081060000 & 1 & BCA11_BCNP_MSH & 0.04 & 1.04 & 0.90 \\
\hline & 02288900 & 3 & 40-Mile_Bend_BCNP_CNL & 3 & 5 & 254721081060000 & 1 & BCA11_BCNP_MSH & 5.14 & 0.82 & 0.92 \\
\hline & 02288900 & 3 & 40-Mile_Bend_BCNP_CNL & 2 & 5 & 260727080590100 & 1 & L28GAP_BCNP_MSH & -0.11 & 0.78 & 0.89 \\
\hline & 02288900 & 3 & 40-Mile_Bend_BCNP_CNL & 1 & 5 & 255806080553500 & 1 & BCA5_BCNP_MSH & -1.69 & 1.10 & 0.88 \\
\hline & 02289019 & 16 & S12BT_ENP_MSTR & 349 & 5 & 02289041 & 15 & S12CT_ENP_MSTR & 0.22 & 0.97 & 0.99 \\
\hline & 02289019 & 16 & S12BT_ENP_MSTR & 348 & 5 & 260007080464401 & 1 & W18_WCA3_MSH & -3.95 & 1.44 & 0.92 \\
\hline & 02289019 & 16 & S12BT_ENP_MSTR & 347 & 5 & 254543080491101 & 15 & S12AT_ENP_MSTR & -0.47 & 1.06 & 0.89 \\
\hline & 02289019 & 24 & S12BH_WCA3_CSTR & 339 & 5 & 254543080491101 & 23 & S12AH_WCA3_CSTR & -0.16 & 1.02 & 1.00 \\
\hline & 02289019 & 24 & S12BH_WCA3_CSTR & 338 & 5 & 02289041 & 3 & S12CH_WCA3_CSTR & 0.22 & 0.98 & 1.00 \\
\hline & 02289019 & 24 & S12BH_WCA3_CSTR & 337 & 5 & 254641080503900 & 1 & S343BH_WCA3_MSTR & 1.40 & 1.02 & 1.00 \\
\hline & 02289041 & 3 & S12CH_WCA3_CSTR & 338 & 5 & 02289019 & 24 & S12BH_WCA3_CSTR & -0.20 & 1.02 & 1.00 \\
\hline & 02289041 & 3 & S12CH_WCA3_CSTR & 337 & 5 & 254543080491101 & 23 & S12AH_WCA3_CSTR & -0.35 & 1.04 & 1.00 \\
\hline & 02289041 & 3 & S12CH_WCA & 336 & 5 & 02289050 & & S333H_WCA3_CSTR & 1.94 & 0.95 & 1.00 \\
\hline
\end{tabular}

Figure 7. Partial view of Gapfill worksheet. Some columns in the application worksheet are hidden so that all essential information can be shown in the figure. 


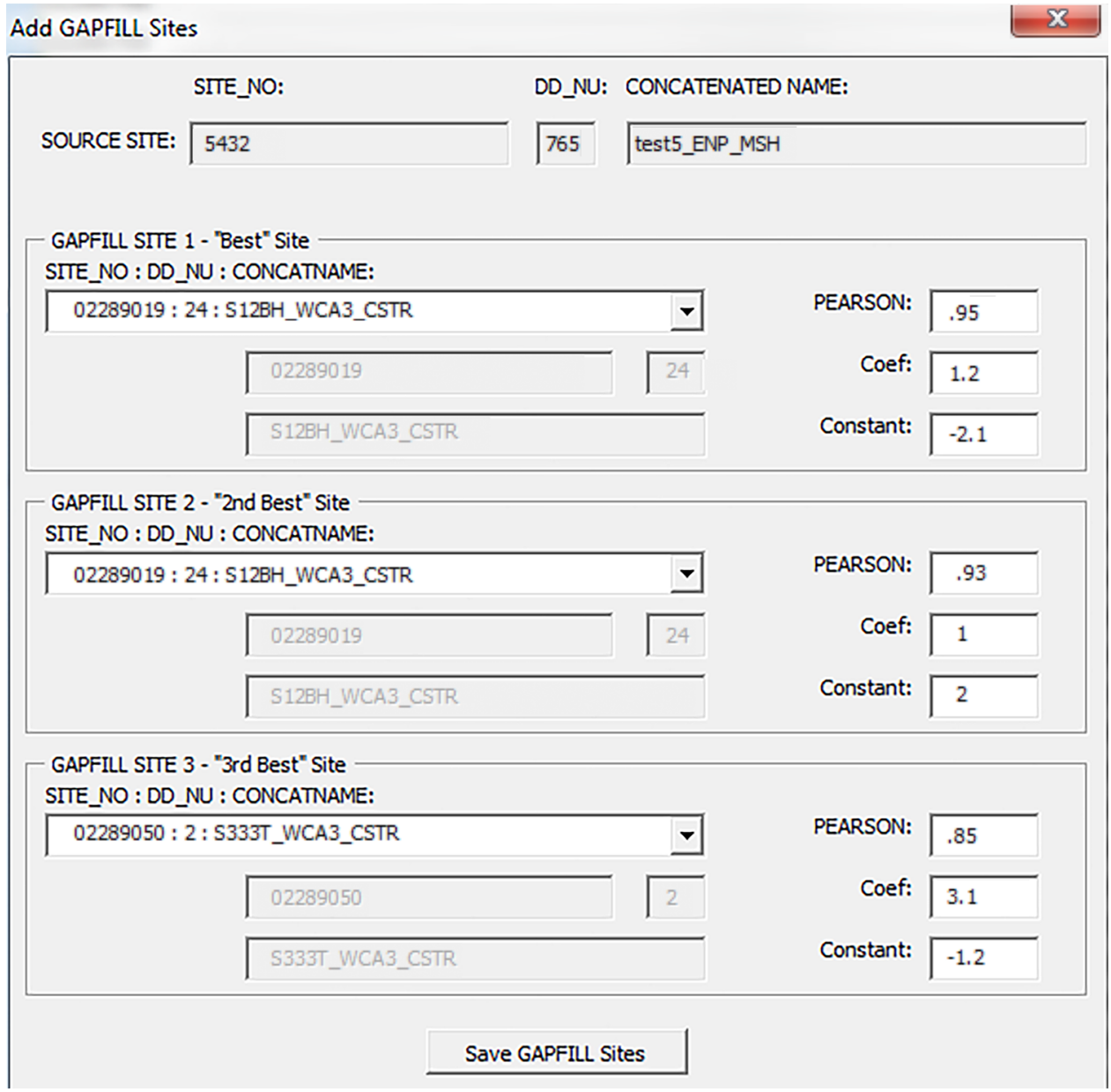

Figure 8. "Add Gapfill Sites" form used to add Gapfill sites for a site. The userform is accessed by double clicking on the SOURCE SITE_NO field in the Gapfill worksheet (fig. 7) . 


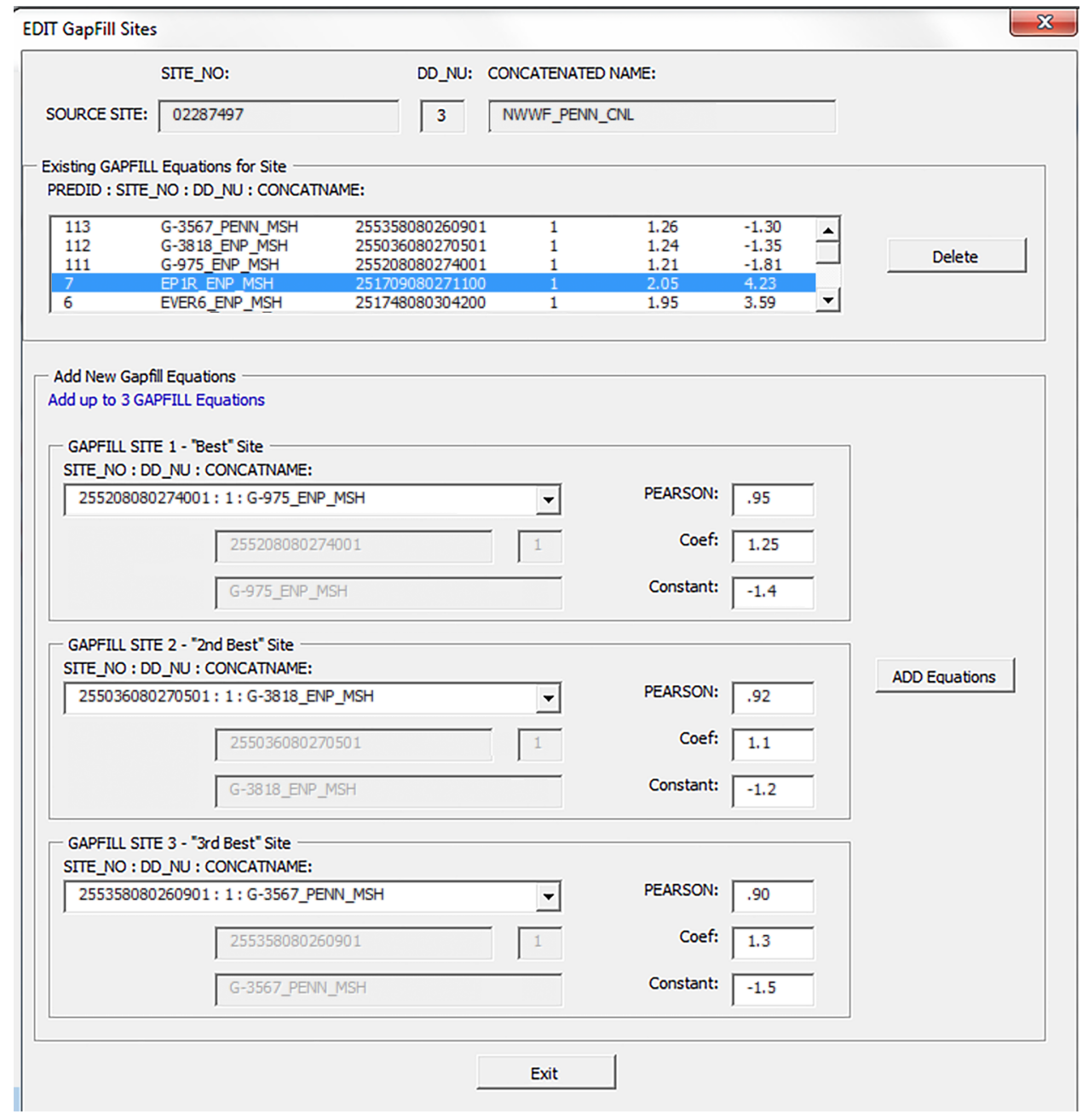

Figure 9. Example of editing Gapfill equations for a site.

Table 1. Format for the UserSiteList worksheet.

[Column header names in bold indicate required fields; max, maximum]

\begin{tabular}{|c|l|l|}
\hline Column & \multicolumn{1}{|c|}{ Column header } & \multicolumn{1}{c|}{ Data required } \\
\hline 1 & station_name & Text, max width 50 characters \\
\hline 2 & station_name_web & Text, max width 50 characters \\
\hline 3 & agency_cd & Text, max width 5 characters \\
\hline 4 & site_no & Text, max width 15 characters \\
\hline 5 & dd_nu & Number, long integer \\
\hline 6 & loc_nu & Number, long integer \\
\hline 7 & loc_nm & Text, max width 25 characters \\
\hline 8 & transmit_id & Text, max width 25 characters \\
\hline 9 & transmit_source & Text, max width 10 characters \\
\hline 10 & station_nm & Text, max width 50 characters \\
\hline 11 & ground_elev_min_88 & Number, single \\
\hline 12 & ground_elev_min_offset & Number, single \\
\hline 13 & ground_elev_avg_88 & Number, single \\
\hline 14 & ground_elev_max_88 & Number, single \\
\hline 15 & datum_change & Number, single \\
\hline
\end{tabular}




\section{Archive Database}

\section{Workbook/Worksheet-ADAM/Control}

Description-Excessively large databases will increase ADAM runtimes. Upon selection of run type (daily, quarterly, or annual), ADAM will check the size of the selected database. If file size is greater than 800 megabytes (MB), ADAM will prompt the user to archive the database (fig. 10A). The message displays in the "Database Maintenance" section of the Control worksheet. Selecting the "Archive" command will open the userform shown in figure 10B. The form displays which data will be removed from the database and placed in the Archives folder. Once archiving is complete, the filename is displayed in the "Data Archived to" field. The user closes the form to proceed with the ADAM run.

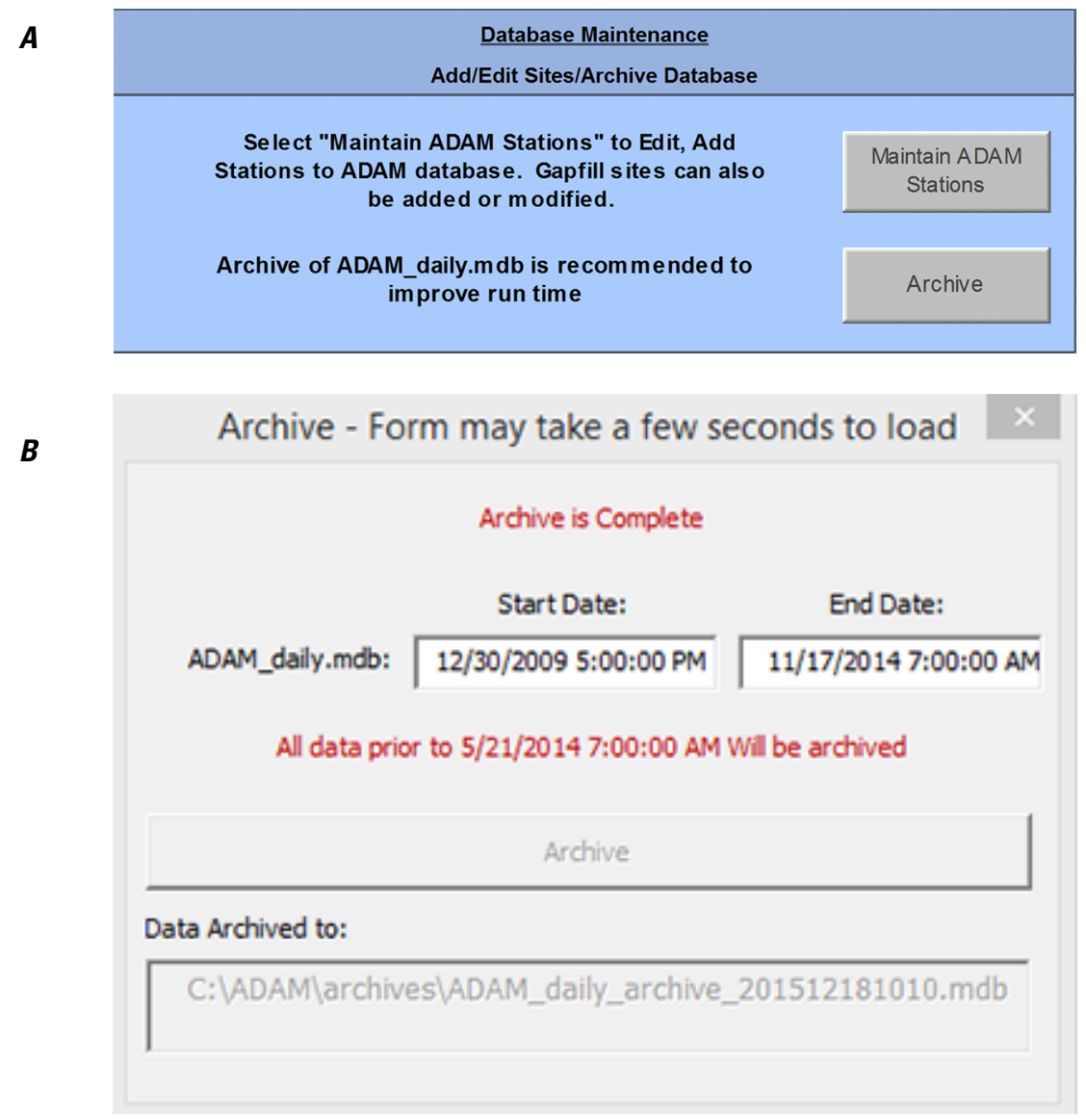

Figure 10. Examples of, $A$, archive prompt displayed in “Database Maintenance" section of the Contro/ worksheet when file size exceeds 800 megabytes and, $B$, userform displayed after selecting "Archive" command in initial prompt.

\section{Filter Settings}

\section{Workbook/Worksheet_-ADAM.xlsm/Filter_Adjustments}

Description - When a new station is added to the ADAMDB the station is also added to [ADAM_SETUP].tbl_FilterSettings (figs. 11-13) with the filterset index set to 0, indicating no filters have been set. A station can have multiple filtersets, but only one set can be active at any given time. This feature allows for the use of different filter settings for different time periods. For example, wet seasons and dry seasons could have different filter settings. Station data can be displayed to aid in setting filter setpoints (fig. 13). There are 14 univariate filters and 1 multivariate filter in current use. Each filter can be enabled or disabled (fig. 11).

Only those filters that are enabled will be used for ADAM analysis. The univariate filters are discussed in the main body of the report. The multivariate filter compares the actual value to the predicted value and trips when the difference between those two values is greater than the user setpoint. 
The Filter_Adjustments worksheet has 3 sections:

1. The top-left section (fig. 11), which contains the current filter settings for a selected station.

2. The bottom section (fig. 12), which is a tabular review of the filter settings for all of the stations. By selecting the appropriate command, the user can display the active filterset for the active stations or all filtersets for active stations. Any stations with no filter assignments will be listed first.

3. The top-right section (fig. 13), which has graphing options to aid in setting the filter setpoints.

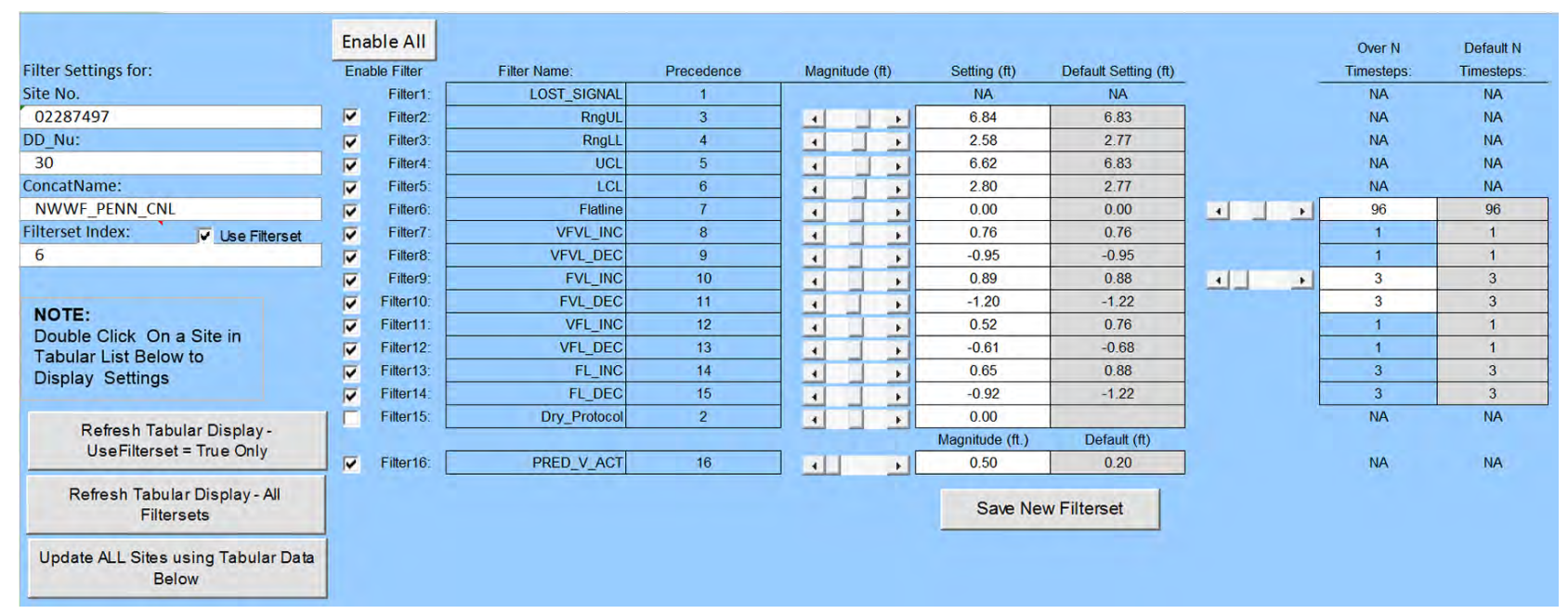

Figure 11. Upper left portion of Filter_Adjustments worksheet. Displays settings for selected Everglades Depth Estimation Network (EDEN) station.

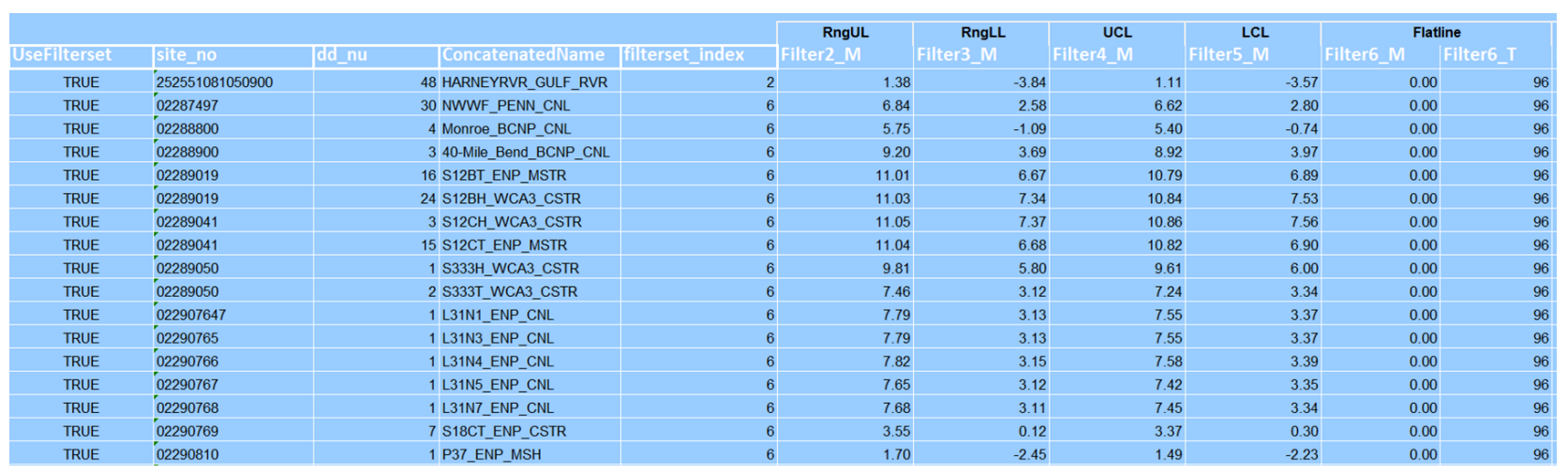

Figure 12. Bottom portion of Filter_Adjustments worksheet. Tabular display of filtersets for active sites. User can either display only the filtersets currently used or display all filtersets for active sites. 


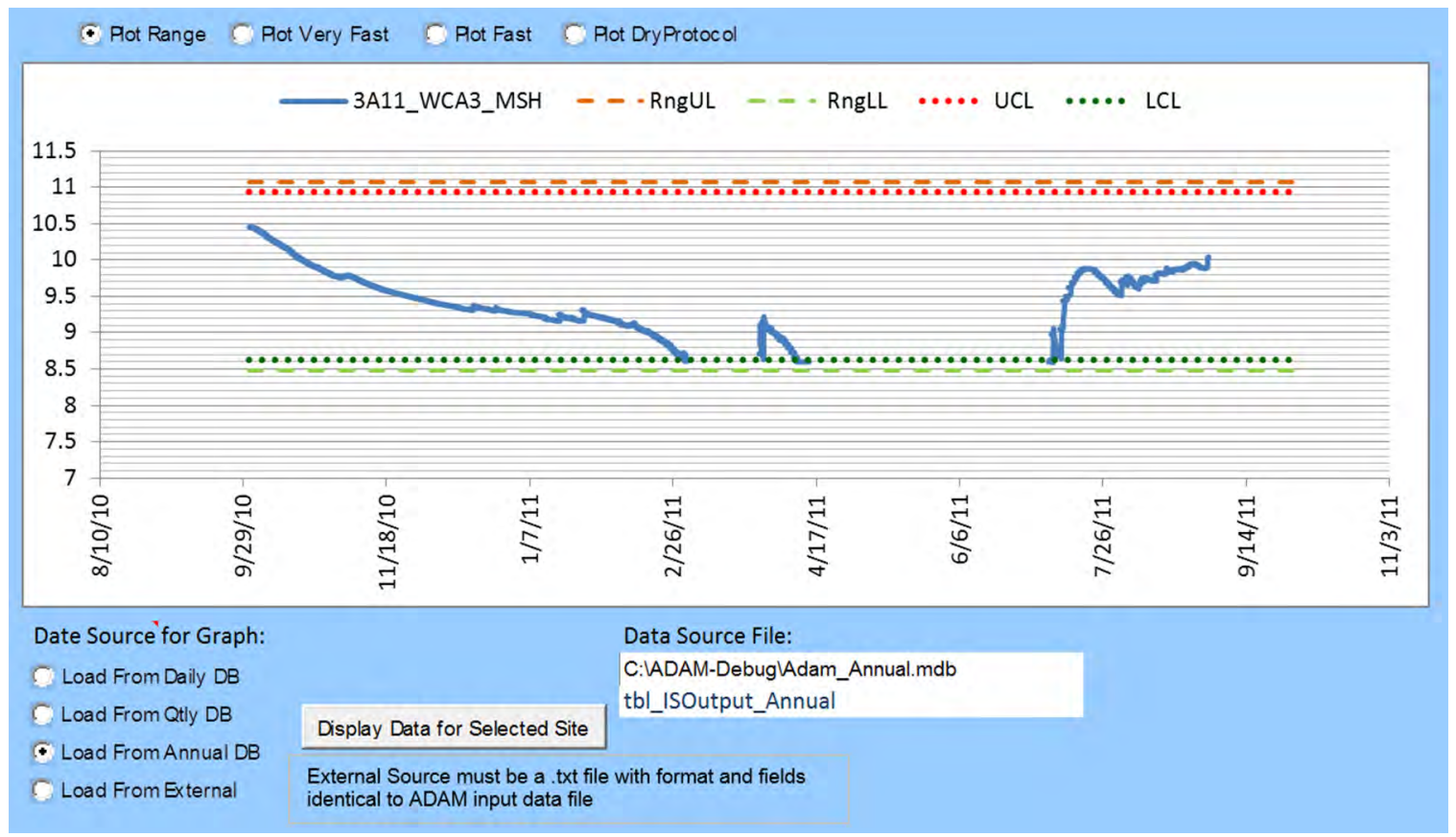

Figure 13. Graphical display of available site data from the selected site. Up to 1 year of data are loaded from the selected site.

\section{Editing Filter Settings for One or a Few Sites}

1. Select a site by double clicking on any cell in the row containing station filter settings (tabular section of the Filter_Adjustments worksheet, fig. 12). This will populate the fields in the upper portion of Filter_Adjustments with the current settings for the active filterset (fig. 11).

2. Make changes using controls in the top section of the worksheet.

3. Save changes by selecting "Save New Filterset." The filterset will be created and made the active filterset. The tabular section will be updated.

\section{Editing Filter Settings for Many Sites}

1. Make any changes needed in the tabular section of worksheet (fig. 12). The user can view the current filter settings for the appropriate formatting requirements.

2. Select "Update ALL Sites using Tabular Data Below" (fig. 11). All filtersets will be updated and filterset_index incremented.

\section{Creating a New Filterset}

Any change to any filter in a filterset requires the creation of a new filterset. When changes are made and saved to [ADAM_ SETUP].tbl_FilterSettings for any station, the created filterset becomes the active set. Once a station is loaded, the user makes any desired changes to the filter settings by using the scroll bars and selecting "Save New Filterset." After saving, a filterset index is assigned and this new filterset becomes the active set. Any previous filtersets for the station become inactive.

\section{Changing Which Filterset Is Active}

1. Select "Refresh Tabular Display - All Filtersets" (fig. 11) to see all defined filtersets for the active stations (fig. 12).

2. Double click site/filterset that requires updating.

3. Check or uncheck the "Use_Filterset" checkbox as needed (fig. 11). The user must ensure that only one filterset is active. 


\section{Displaying Site Data}

The user can display historical data for a given station to aid in setting filter setpoints (fig. 13). Select the data source before selecting the "Display Data for Selected Site" command. If specifying an external file, the format must be identical to the ADAM input file format (Application Input File Format section, herein). Once loaded, the option buttons above the graph can be used to display actual data, the very fast and fast rate-of-change thresholds, and the optional dry protocol threshold. The current filter settings are also displayed. Large datasets will take longer to load than others.

\section{Data Synthesis}

\section{Worksheet-Control}

Description - Certain features of the MLR analysis can be managed by the user by using the "Fill Setup" area of the Control worksheet (fig. 14). The following parameters can be set using the scroll bars:

- Number of stations to include-The number of MLR stations can range from 1 to 5.

- MLR analysis period-The analysis period sets the number of days of data to be used for MLR analysis. The minimum period allowed is 30 days; the maximum period is 366 days.

- Percentage of data required-The required number of days is based on a percentage of the data available for the specified period. For example, for a 100-day period and a setpoint of 75 percent, at least 75 days of concurrent data must be available for the data to be used in the analysis.

- Redo analysis interval (in days) — This is included for the longer quarterly and annual runs and is not applicable for daily runs. A setting of 100 days means that after 100 days, a new set of data will be queried from the ADAMDB for MLR analysis.

- Minimum position of first available gage (nBEST) - Refers to the availability of the "best" station and can be set from "Pos" (position) 1 to 5, where 1 is the best station based on correlation analysis, and 5 would be the fifth best station. This uses the current MLR data but forces ADAM to try and calculate a new regression if the prior prediction did not have the top nBEST (set by user) available. It is recommended that this be set to 1 .

- Restrict SimGage to same conservation area-This option forces the stations selected for MLR analysis to be in the same water conservation area as the station of interest.

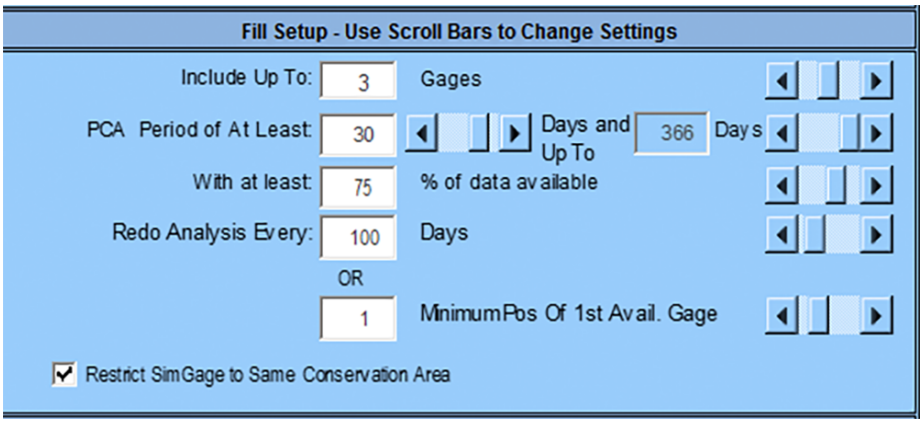

Figure 14. Fill Setup area of the Contro/ worksheet. 


\section{Preliminary Output Logic}

Workbook/Worksheet-ADAM.xlsm/PrelimLogic

Description-The preliminary value output from ADAM is the value used as input to the EDEN database unless overwritten by user review. For each of the 14 univariate filters, the optional "DryProtocol" filter (filter 15), and the multivariate filter (filter 16) the user can select the following options (fig. 15):

- Automatic Override $=$ TRUE - A filter trip results in the actual data being replaced with the predicted value. If no predicted value is available, a NULL will be written to the output.

- Automatic Override = FALSE-A filter trip is treated as a warning flag only. The user has the option to apply an additional check of the actual data:

- User Override Option = NONE — no additional check is made. ADAM will keep the actual value as the output, but the filter trip is noted for closer examination during the manual review process.

- User Override Option = USER CAN SET OVERRIDE RULES-An additional check is applied to the data. For filters $2-15$, the override is applied if the percent difference between the predicted and actual value exceeds the user-set limit. For filter 16, the check is applied to the difference between the predicted value using MLR and the predicted value using Gapfill. If the applicable limit is exceeded, the user can elect to replace the actual data with the predicted or NULL value.

\begin{tabular}{|c|c|c|c|c|c|c|c|c|}
\hline & & & & Override if & 6Error:|(Pred-Actual)/Actual| > & L' & $50 \%$ & \\
\hline & & & & Override if \%Error:|( & redP(A-PredGAP)/PredPCA| > & 1 & $30 \%$ & \\
\hline FilteriD & FILTERTRIP & $\begin{array}{l}\text { Automatic } \\
\text { Override }\end{array}$ & $\begin{array}{l}\text { User Override Options } \\
\text { NONE = Use PREDICTED Value }\end{array}$ & User Override Selection & User Override Rule To Apply & $\begin{array}{l}\text { User Override } \\
\text { Choice }\end{array}$ & $\begin{array}{c}\text { PRELIM VALUE } \\
\text { Automatic Override OR } \\
\text { User Override = FALSE }\end{array}$ & \begin{tabular}{|c|} 
PRELIM VALUE \\
Automatic Override OR \\
User Override $=$ TRUE
\end{tabular} \\
\hline & TRUE & TRUE & NONE & NA & NA & NA & PRED & PRED \\
\hline 2 & TRUE & TRUE & NONE & $\%$ Error - Pred-Act > Setpoint & NONE & PRED & PRED & PRED \\
\hline 3 & TRUE & TRUE & NONE & NONE & NONE & ACTUAL & PRED & PRED \\
\hline 4 & TRUE & FALSE & USER CAN SET OVERRIDE RULES & $\%$ Error - Pred-Act > Setpoint & $\%$ Error - Pred-Act > Setpoint & PRED & ACTUAL & PRED \\
\hline 5 & TRUE & FALSE & USER CAN SET OVERRIDE RULES & \% Error - Pred-Act > Setpoint & $\%$ Error - Pred-Act > Setpoint & PRED & ACTUAL & PRED \\
\hline 6 & TRUE & FALSE & USER CAN SET OVERRIDE RULES & \% Error - Pred-Act > Setpoint & $\%$ Error - Pred-Act > Setpoint & PRED & ACTUAL & PRED \\
\hline 7 & TRUE & TRUE & NONE & $\%$ Error - Pred-Act > Setpoint & NONE & ACTUAL & PRED & PRED \\
\hline 8 & TRUE & TRUE & NONE & $\%$ Error - Pred-Act > Setpoint & NONE & ACTUAL & PRED & PRED \\
\hline 9 & TRUE & TRUE & NONE & $\%$ Error - Pred-Act > Setpoint & NONE & ACTUAL & PRED & PRED \\
\hline 10 & TRUE & TRUE & NONE & $\%$ Error - Pred-Act > Setpoint & NONE & ACTUAL & PRED & PRED \\
\hline 11 & TRUE & FALSE & USER CAN SET OVERRIDE RULES & $\%$ Error - Pred-Act > Setpoint & $\%$ Error - Pred-Act > Setpoint & PRED & ACTUAL & PRED \\
\hline 12 & TRUE & FALSE & USER CAN SET OVERRIDE RULES & $\%$ Error - Pred-Act > Setpoint & $\%$ Error - Pred-Act > Setpoint & PRED & ACTUAL & PRED \\
\hline 13 & TRUE & FALSE & USER CAN SET OVERRIDE RULES & $\%$ Error - Pred-Act $>$ Setpoint & $\%$ Error - Pred-Act > Setpoint & PRED & ACTUAL & PRED \\
\hline 14 & TRUE & FALSE & USER CAN SET OVERRIDE RULES & $\%$ Error - Pred-Act > Setpoint & $\%$ Error - Pred-Act > Setpoint & PRED & ACTUAL & PRED \\
\hline 15 & TRUE & NA & NONE & NA & NA & NA & DryProtocol & NA \\
\hline 16 & TRUE & FALSE & USER CAN SET OVERRIDE RULES & $\%$ Error - Pred-Pred > Setpoint & \% Error - Pred-Pred > Setpoint & ACTUAL & PRED & ACTUAL \\
\hline
\end{tabular}

Figure 15. PrelimLogic worksheet showing logic used by ADAM to set a preliminary value for each station.

\section{Run-Manual}

\section{Workbook/Worksheet-ADAM.xlsm/Control}

Description-ADAM can be run manually from the "Run Setup and Execution" part of the ADAM.xlsm/Control worksheet.

\section{Select run type}

- The user can select daily, quarterly, or annual EDEN validation (fig. 16).

- If the selected database file size is greater than $800 \mathrm{MB}$, the user will be prompted to archive the database before starting the run. Archiving removes all but the most recent 90 days from the database.

\section{Select "Run IS"}

- The user will be prompted to confirm filename and to provide user initials.

- The user will be notified of any active stations having no univariate filter settings and is given the option to proceed without the filter settings or cancel the run.

Once the run is complete and all data are saved to the output database, the status "Run Complete" is displayed in the "Status of Current Run" field (fig. 17). 


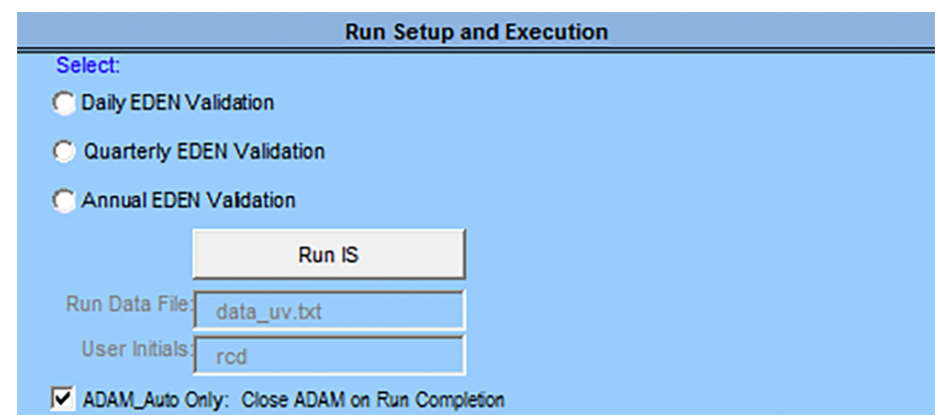

Figure 16. Control worksheet "Run Setup and Execution" controls.

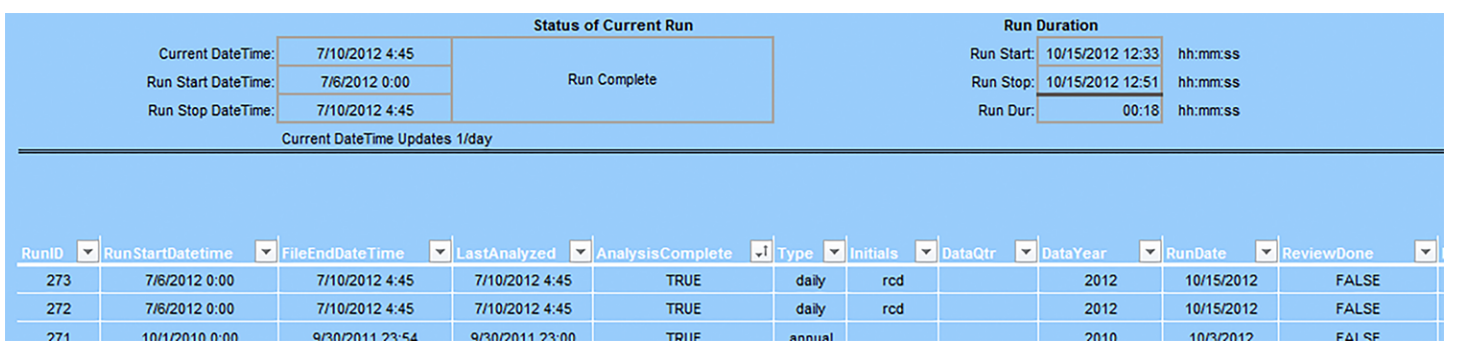

Figure 17. Contro/ worksheet—run progress and tabular run history.

\section{Run-Auto}

Description_ADAM can be set to run automatically for daily runs by creating an ADAM_Auto.xlsm workbook. Automatic runs are not available for quarterly or annual runs. To create a self-running workbook, the user does the following:

1. Open ADAM.xlsm and set up the inputfiles data path to point to the directory that will contain the daily file (discussed in the Set File Paths section, herein).

2. Enter the name of the daily file in the "Run Data File" textbox cell of the "Run Setup and Execution" part of the Control worksheet (fig. 16).

3. Enter initials in "User Initials" textbox.

4. Select "Close ADAM on Run Completion" checkbox, if desired.

5. Save workbook as "ADAM_Auto.xlsm".

6. Create a scheduled task to open ADAM_Auto.xlsm at a specified time each day.

When ADAM_Auto.xlsm is opened, it will do the following:

1. Analyze the data contained in the input data file specified during setup.

2. Export the IS_Output table for the run as a .txt file. (The table is exported to the directory specified as the outputfiles directory during ADAM setup.)

3. Save and close itself and exit Excel if no other workbooks are open.

\section{Run Review}

Run reviews use the ADAM_Review.xlsm (ADAM_REVIEW) application. The source data for ADAM_REVIEW are in the run output file (ADAM_Runnn.mdb). Station reviews are not required; however, if a review is not completed, the ADAMassigned preliminary value (PrelimValue) is used as output for the EDEN database. (Fig. 15 shows the logic used in assigning a preliminary value.) Once the run review is complete, the results can be saved to the appropriate database ("daily," "qtly," or "annual"), referred to as the archival database. 


\section{Selecting a Review}

\section{Workbook/Worksheet_-ADAM_Review.xlsm/Control \\ Starting a Review-}

1. Verify file paths by making sure that the file paths are correct (fig. 18). If necessary, update using the "Set DB Path" and "Set Output Data Path" commands.

2. Select the RunID by using the drop-down list "RunID Selection From List" (fig. 19). The list is populated by all runs listed in the ADAM_Setup.mdb file. Users can also manually enter a run number (fig. 20) if reviewing a run without access to the source ADAM_Setup.mdb file. Not all functions are available in this case.

3. Select the days to be included in filter trip listings. The "1st Day in Review" entry (fig. 19) refers to the first day that will be included in generating a listing of stations with filter trips. This is normally the same as the "Run Start Date" entry but can be adjusted by as much as 30 days. The "\# Days in Review" entry indicates the number of days to include in the listing of stations with filter trips and can range from 1 to 7 days or "ALL" days in the review file. For quarterly and annual runs, this field should be set to "ALL" so that all data are included in the review. These settings will only affect the stations populated in the filter trip listings contained in the Review_Chart worksheet and allow the reviewer to focus on the days of interest.

4. Select "Review" to load worksheets Review_Chart and Review_Tab. Review_Chart provides a graphical display of the run data. Review_Tab provides a tabular display of the run data and allows the reviewer to review the run data, estimate any missing or bad data, and save the reviewed values to the run output file. Once all reviewing is complete, the results can be saved to the archival database.

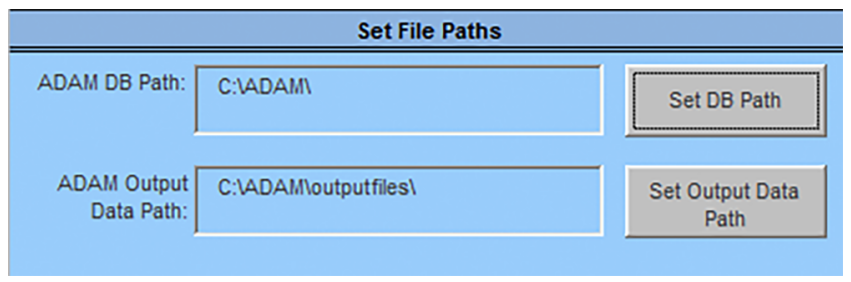

Figure 18. Section of the Contro/ worksheet used to set file paths.

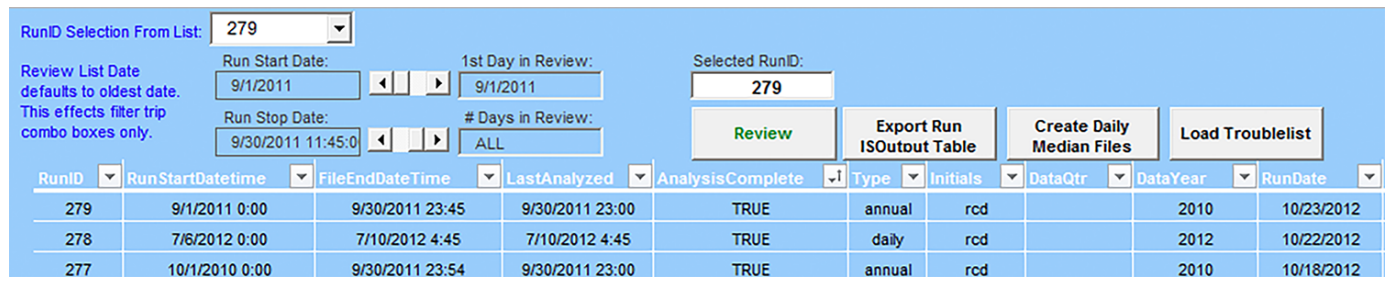

Figure 19. Section of the Contro/ worksheet used to select run for review. Includes a tabular listing of all runs in the ADAM_Setup.mdb.

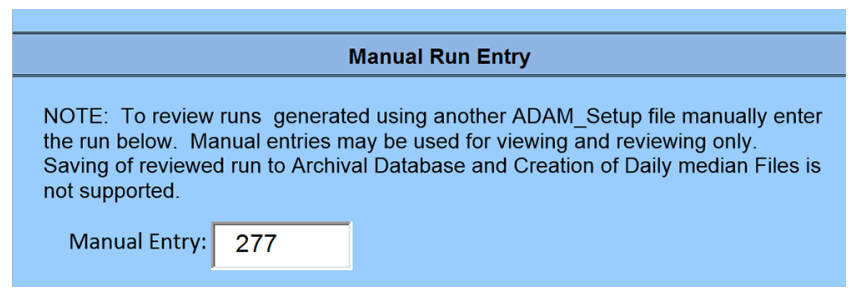

Figure 20. Section of the Contro/ worksheet used to manually enter a run for review. This is only used when the source ADAM_Setup.mdb and ADAM archival database files are not available. Not all features of ADAM_REVIEW are available. 


\section{The Review Process}

\section{Selecting a Site}

One of three drop-down combo boxes are used to select the station for review or one of three spin buttons can be used to scroll through the lists (fig. 21). The selection options are

- Rev Site Any Flt - Any site that tripped any filter during the run.

- Rev Site Flt 1 - Any site that had missing data during the run.

- Rev Site - All—_All sites in [ADAM_SETUP].tbl_StationList.

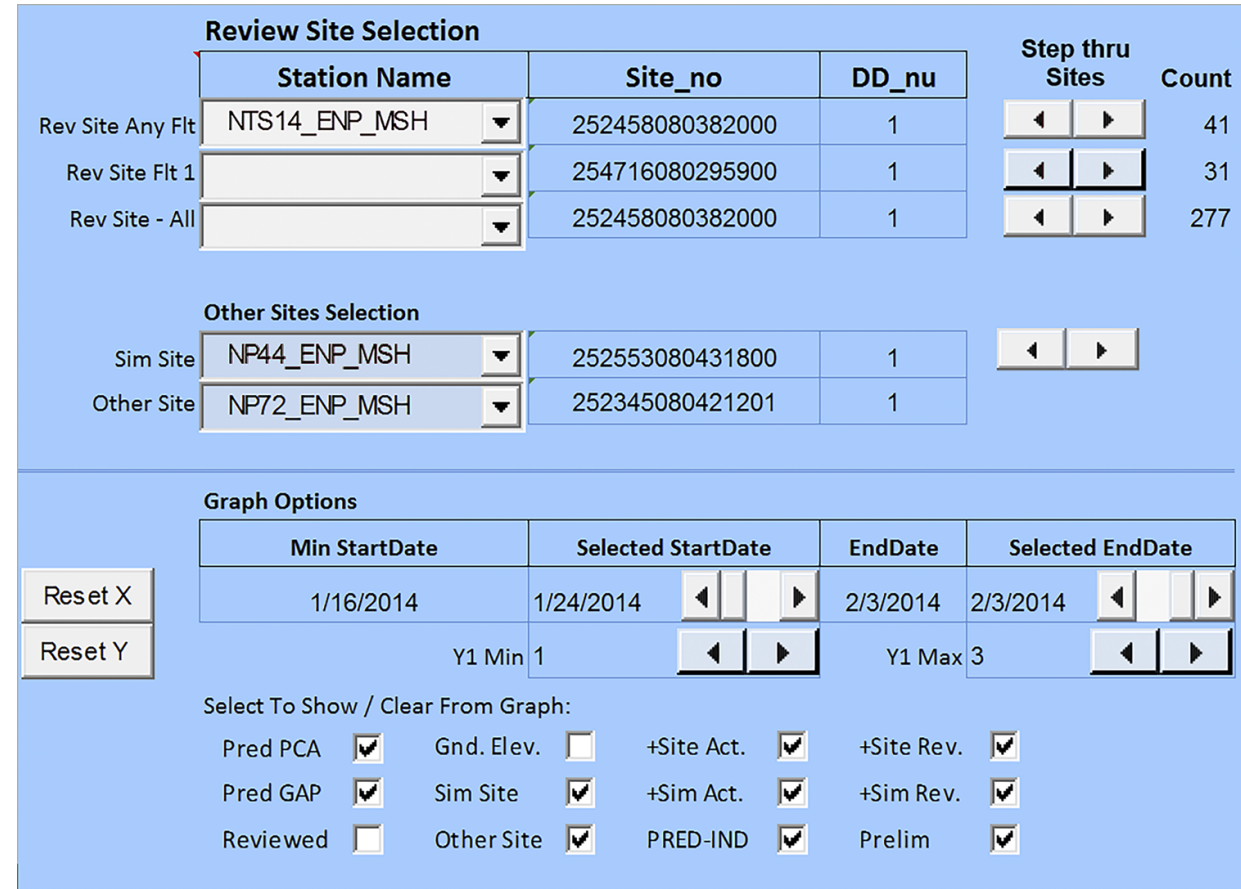

\section{EXPLANATION}

$\begin{array}{cl}\text { PCA } & \text { Multivariate linear regression estimate } \\ \text { SLR } & \text { Simple linear regression estimate } \\ \text { Reviewed } & \text { Previously reviewed data } \\ \text { Similar Site } & \begin{array}{c}\text { Previously reviewed data and current } \\ \text { data for a similar station }\end{array} \\ \text { Other Site } & \begin{array}{c}\text { Previously reviewed data and current data } \\ \text { for an additional similar station }\end{array} \\ \text { Filter ID } & \text { Filter trip identification number } \\ \text { PRED-IND } & \text { Predictor indicator }\end{array}$

Figure 21. Screen capture showing Review_Chart worksheet—site selection and graph setup. 


\section{Configuring the Graph}

The graph will always display the actual data for the selected station as well as any filter trips. In addition, the following can be selected or deselected for display (fig. 21):

- Pred PCA - Predicted values generated using MLR. If MLR analysis cannot be performed, this will display the Gapfill prediction.

- Pred GAP — Predicted values generated using Gapfill equation.

- Reviewed-Reviewed values.

- Gnd. Elev.-Ground elevation for the station.

- Sim Site - Values from a similar site used in making predictions for the selected review site during this run. This feature is enabled by selecting a site in the "Sim Site" drop-down combo box and checking the "Sim Site" checkbox (fig. 21)

- Other Site-Any site in [ADAM_SETUP].tbl_StationList. The site can be selected by using the "Other Site" drop-down combo box and checking "Other Site" checkbox (fig. 21).

- +Site Act. - Fourteen days of actual site data preceding the run date. Control is disabled if data are not available.

- +Sim Act.-Fourteen days of actual site data from a similar site preceding the run date. Control is disabled if data are not available.

- PRED-IND - Scaled predictor ID to visualize change in predictor equation.

- +Site Rev.-Fourteen days of reviewed site data preceding the run date. Control is disabled if data are not available.

- +Sim Rev.-Fourteen days of reviewed data from a similar site preceding the run date. Control is disabled if data are not available.

- Prelim - Preliminary output set by ADAM. This is the value used as input to the EDEN database unless overwritten during user review.

The $\mathrm{X}$ and $\mathrm{Y}$ axes can be changed using the controls found under "Graph Options" (fig. 21). Once changed, one or both axes can be reset to their full range using "Reset X" and (or) "Reset Y." Figure 22 shows a sample graphical display.

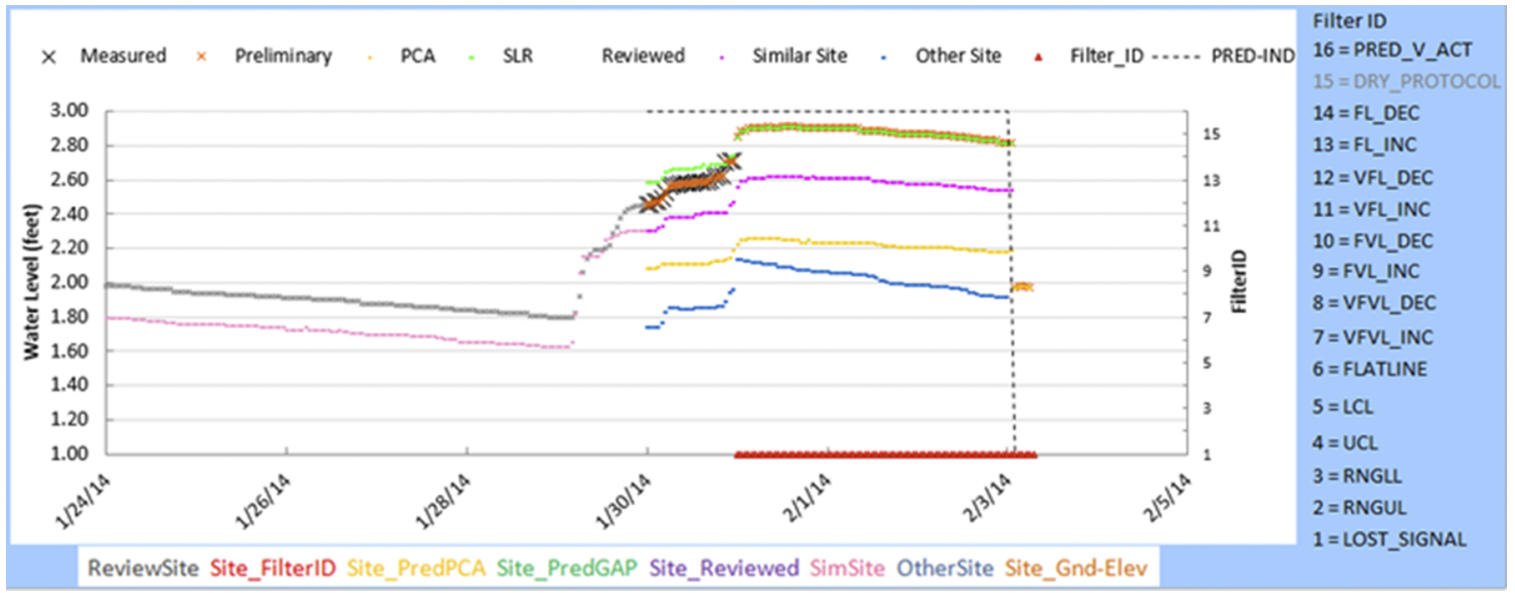

\section{EXPLANATION}

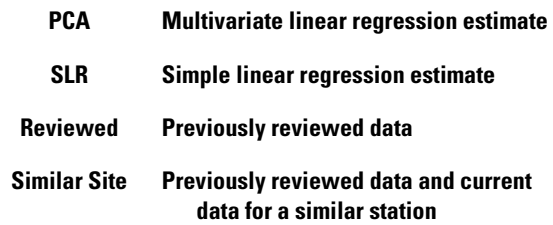
Other Site Previously reviewed data and current data for an additional similar station

Filter_ID Filter trip identification number

PRED-IND Predictor indicator data for a similar station

Figure 22. Sample graph display generated using the Review_Chart worksheet. 


\section{Tabular Review}

The Review_Tab worksheet displays a tabular list of data available for the review of a selected site (fig. 23). The following are included in the tabular data:

- date_tm-Date and time.

- ActualValue - Value read in from input file.

- FilteredValue - Value after univariate filtering is applied. FilteredValue = ActualValue if no filter is tripped; FilteredValue $=$ "Null" if filter is tripped.

- PredValue - Predicted using MLR analysis if available or by Gapfill equation if MLR is not available.

- GapFillPred-Predicted using the "best" available Gapfill equation.

- datum_change —Value stored in [ADAM_SETUP].tbl_StationList. Datum_change values are included in the PrelimValue.

- PrelimValue - Preliminary value selected by ADAM logic. If data have not been reviewed, this value is written to the daily output files.

- RevValue - Reviewed value. If this value is not "Null," the value is written to the daily output files.

- Manual Entry - Allows the user to enter a value to use as the "RevValue."

- Start and Stop columns - Supports calculated manual data corrections. The user double-clicks the appropriate row in these columns to select the interval for any shifts, interpolations, or holds. The "Data Correction" drop-down box is used to select the operation (fig. 23). Finally, selecting the command "Perform Data Correction" will populate the manual entry column with appropriate data.

- Review Selection - Users set the reviewed values using the "For All Values Use" drop-down combo box or making individual selections in the "Review Selection" column (fig. 23). If a selection is made for the "For All Values Use" combo box, the same datatype choice will be selected for the RevValue for all timestamps. Alternatively, the datatype choice can be individually set for each time step; choices include the following: preliminary value set by ADAM, actual value, filtered value, predicted value, Gapfill value, manual entry, or keep prior review.

- Support Data - The data to the right of the "Review Selection" column within the Review_Tab worksheet are highlighted in gray and provided to support the review process.

- Quick look at prediction statistics - Double-clicking on a cell in the "PredValue" or "GapFillPred" columns will display statistics for the prediction, including the coefficient of determination $\left(\mathrm{R}^{2}\right)$ and station(s) used in the prediction (fig. 24).

\begin{tabular}{|c|c|c|c|c|c|c|c|c|c|c|c|c|c|}
\hline \multicolumn{6}{|l|}{ RunID } & & & & \multicolumn{5}{|l|}{ Data Correction: } \\
\hline 779 & \multirow{3}{*}{\multicolumn{2}{|c|}{$\begin{array}{l}\text { Save Reviewed Data for } \\
\text { Selected Site }\end{array}$}} & \multirow{3}{*}{\multicolumn{3}{|c|}{$\begin{array}{l}\text { Run Review Complete - Load } \\
\text { Review in Archival Database } \\
\text { and Export ISOutput Text File }\end{array}$}} & \multirow{5}{*}{\multicolumn{3}{|c|}{$\begin{array}{l}\text { Double click in the appropriate } \\
\text { rows in the the "Start" and "Stop" } \\
\text { Columns to select interval for } \\
\text { Shift, Interpolation, or Hold }\end{array}$}} & \multicolumn{2}{|l|}{ Actual Hold } & \multirow[t]{4}{*}{$\nabla$} & \multirow{4}{*}{\multicolumn{2}{|c|}{$\begin{array}{l}\text { Select Review Value for Each } \\
\text { Date Time by setting each Row } \\
\text { OR Select Same Choice for All } \\
\text { by Selecting Option Below } \\
\text { For All Values Use }\end{array}$}} \\
\hline Reviewer Initials & & & & & & & & & & & & & \\
\hline MDP & & & & & & & & & \multirow{3}{*}{\multicolumn{3}{|c|}{ Perform Data Correction }} & & \\
\hline Concatenated Name & \multicolumn{2}{|r|}{ RecordCount } & & & & & & & & & & & \\
\hline NTS14_ENP_MSH & & 104 & & & & & & & & & & \multicolumn{2}{|l|}{ Use Individual Reviews } \\
\hline date_tm & ActualValue & FilteredValue & PredValue & GapFillPred & datum_ & change | & PrelimValue & RevValue & Manual Entry & Start & Stop & Review Selection & Prelim \\
\hline $1 / 30 / 20140: 00$ & 2.46 & 2.46 & 2.09 & 2.59 & $c$ & 0 & 2.46 & 2.46 & & & & Keep Prior Review & \\
\hline $1 / 30 / 20141: 00$ & 2.46 & 2.46 & 2.09 & 2.59 & c & 0 & 2.46 & 2.46 & & & & Keep Prior Review & \\
\hline $1 / 30 / 20142: 00$ & 2.47 & 2.47 & 2.09 & 2.59 & 0 & 0 & 2.47 & 2.47 & & & & Keep Prior Review & \\
\hline $1 / 30 / 20143: 00$ & 2.47 & 2.47 & 2.10 & 2.59 & c & 0 & 2.47 & 2.47 & & & & Keep Prior Review & \\
\hline $1 / 30 / 20144: 00$ & 2.49 & 2.49 & 2.11 & 2.61 & c & 0 & 2.49 & 2.49 & & & & Keep Prior Review & \\
\hline $1 / 30 / 20145: 00$ & 2.53 & 2.53 & 2.11 & 2.65 & c & 0 & 2.53 & 2.53 & & & & Keep Prior Review & \\
\hline $1 / 30 / 20146: 00$ & 2.56 & 2.56 & 2.11 & 2.66 & c & 0 & 2.56 & 2.56 & & & & Keep Prior Review & \\
\hline $1 / 30 / 20147: 00$ & 2.57 & 2.57 & 2.11 & 2.67 & 0 & 0 & 2.57 & 2.57 & & & & Keep Prior Review & \\
\hline $1 / 30 / 20148: 00$ & 2.57 & 2.57 & 2.11 & 2.67 & c & 0 & 2.57 & 2.57 & & & & Keep Prior Review & \\
\hline 1/30/2014 9:00 & 2.58 & 2.58 & 2.11 & 2.67 & c & 0 & 2.58 & 2.58 & & & & Keep Prior Review & \\
\hline $1 / 30 / 201410: 00$ & 2.58 & 2.58 & 2.11 & 2.67 & c & 0 & 2.58 & 2.58 & & & & Keep Prior Review & \\
\hline
\end{tabular}

Figure 23. Review_Tab worksheet—partial view. 


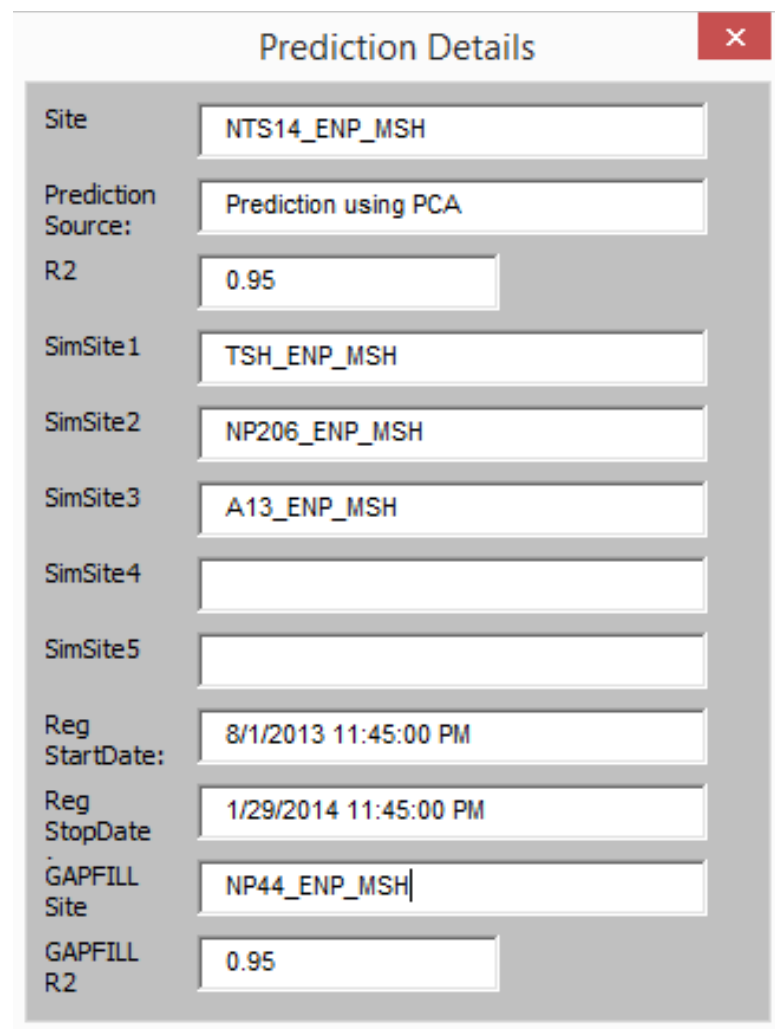

Figure 24. Screen capture showing display of prediction details accessed from the Review_Tab worksheet. Double clicking on a cell in the "PredValue" or "GapFillPred" columns in the worksheet will display the coefficient of determination $\left(\mathrm{R}^{2}\right)$ for the regression and the sites used in the prediction.

\section{Steps to Review a Site and Save Results}

1. Select a site using combo boxes in the Review_Chart worksheet (fig. 21).

2. Make a review selection in the Review_Tab worksheet (fig. 23).

3. Save the reviewed site selection by clicking "Save Reviewed Data for Selected Site" (fig. 23). Review is not required. If no review is made, the PrelimValue will be used for the daily output files. Once saved, the reviewed value can be displayed on the graph for visual confirmation by checking the "Reviewed" box on the Review_Chart worksheet (fig. 21).

4. Save review to the archival database after completion of all reviews by selecting the "Run Review Complete-Load Review in Archival..." command (fig. 23). This process may take several minutes. This command will also export the data to the ISOutput Text File.

\section{Load and Review TroubleList}

Workbook/Worksheet_ADAM_Review.xlsm/Control

Description-The list of filter trips for the current run can be reviewed by selecting the "Load Troublelist" command in the Control worksheet (fig. 19). Once in the Current_TroubleList worksheet (fig. 25), the user can list all of the current filter trips or select a subset using the "Load Filter" drop-down list. The user can return to the Control worksheet by selecting the "Return to Control WS" command (fig. 25). 


\begin{tabular}{|c|c|c|c|c|c|c|c|c|}
\hline station_name & site_no & dd_nu. & filterid. & concatenatedname & Column1 & Load Filter & RunID & \multirow{2}{*}{$\begin{array}{l}\text { Load Current } \\
\text { Trouble List }\end{array}$} \\
\hline $3 \mathrm{~A} 10+$ & 261645080442400 & 1 & 1 & 3A10_WCA3_MSH & & 1 & 779 & \\
\hline $3 \mathrm{~A} 11+$ & 261305080443800 & 1 & 1 & 3A11_WCA3_MSH & \multirow{6}{*}{$\begin{array}{l}1 \\
2 \\
3 \\
4 \\
5 \\
6 \\
7 \\
8\end{array}$} & & $\wedge$ & \\
\hline $3 A 12+$ & 261008080403300 & 1 & 1 & 3A12_WCA3_MSH & & & & \multirow{2}{*}{$\begin{array}{c}\text { Return to Control } \\
\text { WS }\end{array}$} \\
\hline 3AN1W1+ & 261116080442500 & 1 & 1 & 3AN1W1_WCA3_MSH & & & & \\
\hline 3ANE + & 261643080361800 & 1 & 1 & 3ANE_WCA3_MSH & & & & \\
\hline $3 A N E+G W$ & 261643080361801 & 1 & 1 & 3ANEGW_WCA3_MSH & & & $\checkmark$ & \\
\hline 3ANW+ + & 261708080463300 & 1 & 1 & 3ANW_WCA3_MSH & & & & \\
\hline $3 A N W+G W$ & 261708080463301 & 1 & 1 & 3ANWGW_WCA3_MSH & & & & \\
\hline $3 A S+$ & 260500080410400 & 1 & 1 & 3AS_WCA3_MSH & & & & \\
\hline 3AS3W1+ & 255126080461600 & 1 & 1 & 3AS3W1_WCA3_MSH & & & & \\
\hline 3ASW+ & 255923080501000 & 1 & 1 & 3ASW_WCA3_MSH & & & & \\
\hline 3BS1W1+ & 254649080304100 & 1 & 1 & 3BS1W1_WCA3_MSH & & & & \\
\hline 3B-SE+ + & 254716080295900 & 1 & 1 & 3BSE_WCA3_MSH & & & & \\
\hline
\end{tabular}

Figure 25. Screen capture showing Current_TroubleListworksheet. Listing of all sites exhibiting filter trips for the current run. User can select all or by filter trip.

\section{Create Daily Median Files}

Workbook/Worksheet_ADAM_Review.xlsm/Control

Description -Writes the daily median files required by the EDEN surface-water model to the Outputfiles directory (fig. 26). Select the "Create Daily Median Files" command to create files.

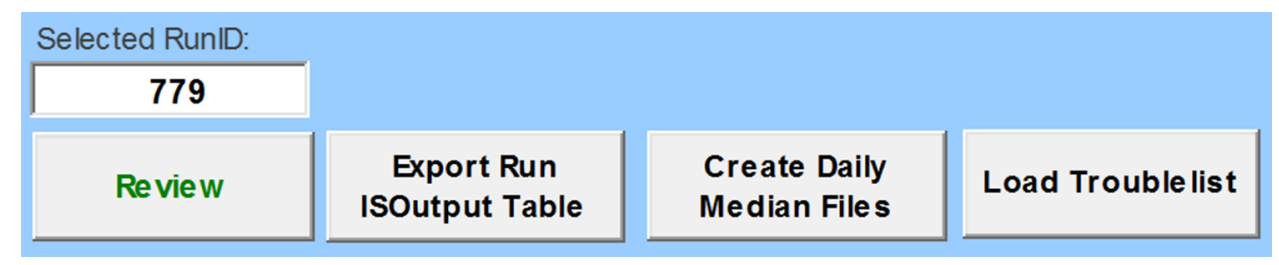

Figure 26. Screen capture showing Controlworksheet commands used to generate daily median files and export data. Selecting the "Export Run ISOutput Table" command button will export the data to a text file.

\section{Export ISOutput as ASCII text file}

Workbook/Worksheet_ADAM_Review.xlsm/Control or Review_Tab

Description-Exports ISOutput ASCII text file to outputfiles directory using command button in the Review_Tab or Control worksheets (figs. 23 and 26, respectively). 


\section{Application Input File Format}

Data Input File - ASCII text file (tab delimited) containing the following columns with header on first row (table 2)

Table 2. Format of ADAM data input file.

[ADAM, Automated Data Assurance and Management; max, maximum; MM/DD/YYYY, month, day, year; HH:MM:SS, hour, minute, second]

\begin{tabular}{|c|l|l|}
\hline Column & \multicolumn{1}{|c|}{ Field name } & \multicolumn{1}{c|}{ Type } \\
\hline 1 & agency_cd & Text, max width 5 characters \\
\hline 2 & site_no & Text, max width 15 characters \\
\hline 3 & dd_nu & Number, long integer, max width 4 characters \\
\hline 4 & parameter_cd & Text, max width 5 characters \\
\hline 5 & UVTYPE & Text, max width 2 characters \\
\hline 6 & date_tm & DateTime, format MM/DD/YYYY HH:MM:SS \\
\hline 7 & TZCD & Text, max width 3 characters \\
\hline 8 & VALUE & Number, decimal, max width 5 characters \\
\hline 9 & PRECISION & Number, long integer, max width 1 character \\
\hline 10 & REMARK & Text, max width 10 characters \\
\hline 11 & FLAGS & Text, max width 10 characters \\
\hline 12 & QA & Text, max width 10 characters \\
\hline
\end{tabular}

\section{Application User Worksheets}

The following worksheets are used or referenced by an ADAM user (tables 3 and 4). All other ADAM worksheets are for ADAM use only.

Table 3. ADAM worksheets that are accessed for setting up or completing an ADAM run.

[ADAM, Automated Data Assurance and Management]

\begin{tabular}{|l|l|}
\hline \multicolumn{1}{|c|}{$\begin{array}{c}\text { ADAM.xIsm worksheet } \\
\text { name }\end{array}$} & \multicolumn{1}{c|}{ Description } \\
\hline About & Lists current ADAM version and contact information. \\
\hline Control & Used to setup and run ADAM. \\
\hline Filter_Adjustments & Used to set/modify filter settings for selected station(s). \\
\hline ADAMSiteList & Used to add or modify data for selected station(s). \\
\hline UserSiteList & Used to upload station information for new sites to ADAM_SETUP database. \\
\hline Gapfill & Used to manage Gapfill sites. \\
\hline RevisionHistory & Lists the history of modifications/updates to ADAM. \\
\hline
\end{tabular}

Table 4. ADAM worksheets that are accessed for completing an ADAM review.

[ADAM, Automated Data Assurance and Management]

\begin{tabular}{|l|l|}
\hline \multicolumn{1}{|c|}{$\begin{array}{c}\text { ADAM_Review.xIsm } \\
\text { worksheet name }\end{array}$} & \multicolumn{1}{c|}{ Description } \\
\hline About & Lists current ADAM_REVIEW version and contact information. \\
\hline Control & Used to setup and start a run review. \\
\hline Review_Tab & $\begin{array}{l}\text { Tabular review of a selected run. Used to select reviewed data and save site review } \\
\text { to run database. Upon review completion, reviewed data can be saved to archival } \\
\text { database. }\end{array}$ \\
\hline Review_Chart & Used to select site to be reviewed. Graphical display of site data and predictions. \\
\hline Current_TroubleList & List of all filter trips for a selected run. Can list all or select by FilterID. \\
\hline RevisionHistory & Lists the history of modifications/updates to ADAM_REVIEW. \\
\hline
\end{tabular}




\section{ADAMDB Table Structure}

Database-ADAM_Setup.mdb

Use-Stores the list of gaging stations to be analyzed by ADAM, filter settings used for each station, ADAM run ids, MLR and Gapfill prediction equations and status notes for the predictions, preliminary values, and reviewed values (table 5).

Table 5. ADAM_Setup.mdb tables and queries required by ADAM.

[ADAM, Automated Data Assurance and Management; $\mathrm{R}^{2}$, coefficient of determination; PCA, principal component analysis]

\begin{tabular}{|l|l|}
\hline \multicolumn{1}{|c|}{ Table(tbl) or query(qry) name } & \multicolumn{1}{c|}{ Description } \\
\hline tbl_FilterDefinitions & Definitions for each of the filters used. \\
\hline tbl_FilterSettings & Filter setpoints/thresholds used for each station. \\
\hline tbl_PredEquations & $\begin{array}{l}\text { Prediction details including sites used, regression coefficients, } \mathrm{R}^{2}, \text { and } \\
\text { PCA eigenvectors and eigenvalues. }\end{array}$ \\
\hline tbl_RunIDs & Listing of IDs for each ADAM run. \\
\hline tbl_PredNotes & Descriptive status for prediction. \\
\hline tbl_PrelimNotes & Descriptive status for ADAM selected preliminary value. \\
\hline tbl_RevNotes & Descriptive status of user-reviewed value. \\
\hline tbl_StationList & List of stations used by ADAM. \\
\hline tbl_VersionHistory & Lists the history of modifications and (or) updates to database format. \\
\hline
\end{tabular}

Database-ADAM_type.mdb (type = daily, qtly or annual)

Use-Stores up to two years of historical data and current data to be analyzed by ADAM (table 6). There is a database for each of the three run types: daily, quarterly, and annual.

Table 6. ADAM_type.mdb tables and queries required by ADAM.

[ADAM, Automated Data Assurance and Management. The word "type" in file, table, or query name indicates whether object is part of a daily (daily), quarterly (qtly), or annual (annual) run]

\begin{tabular}{|c|c|}
\hline Table(tbl) or query(qry) name & Description \\
\hline tbl_ISOutput_type & $\begin{array}{l}\text { Stores measured ("actual") data and results of ADAM } \\
\text { analysis. }\end{array}$ \\
\hline qry_ISOutput_type & $\begin{array}{l}\text { Used by ADAM application to merge data from tables } \\
\text { tbl_ISOutput, tbl_StationList, tbl_PredEquations, } \\
\text { tbl_PredNotes, tbl_RevNotes, and tbl_PrelimNotes. }\end{array}$ \\
\hline tbl_VersionHistory & $\begin{array}{l}\text { Lists the history of modifications and (or) updates to } \\
\text { database format. }\end{array}$ \\
\hline \multicolumn{2}{|c|}{ Tables linked to ADAM_Setup.mdb } \\
\hline tbl_PredEquations & \multirow{6}{*}{$\begin{array}{l}\text { Tables are imported from the ADAM_SETUP database } \\
\text { for use by ADAM. }\end{array}$} \\
\hline tbl_PredNotes & \\
\hline tbl_PrelimNotes & \\
\hline tbl_RevNotes & \\
\hline tbl_StationList & \\
\hline tbl_RunIDs & \\
\hline
\end{tabular}


Database-ADAM_Import.mdb

Use-Temporarily stores data imported for analysis (table 7).

Table 7. ADAM_Import.mdb tables and queries required by ADAM.

[ADAM, Automated Data Assurance and Management. The word "type" in file, table, or query name indicates whether object is part of a daily (daily), quarterly (qtly), or annual (annual) run]

\begin{tabular}{|c|c|}
\hline Table name & Description \\
\hline tbl_imported_type & $\begin{array}{l}\text { Data imported from input text file (data_uv.txt). Data are deleted after } \\
\text { they are analyzed. }\end{array}$ \\
\hline tbl_unreaddata_type & $\begin{array}{l}\text { Upon run completion this table is populated with all data not analyzed } \\
\text { by ADAM. Data populating this table can result from the data hav- } \\
\text { ing a timestamp that is not on hourly or 15-minute timestamps or the } \\
\text { data are for a site that is not listed in the ADAM_SETUP tbl_Sta- } \\
\text { tionList table. }\end{array}$ \\
\hline tbl_workingdata_type & $\begin{array}{l}\text { This table is a small subset of tbl_imported_type table and is used to } \\
\text { improve runtime performance. }\end{array}$ \\
\hline dataforfilterplotting & $\begin{array}{l}\text { Data imported from user-specified .txt file to support filter setting } \\
\text { adjustments. These data must have the same format as the input text } \\
\text { file (data_uv.txt). }\end{array}$ \\
\hline tbl_VersionHistory & Lists the history of modifications and (or) updates to database format. \\
\hline
\end{tabular}

Database-ADAM_Runnn.mdb (where $n n=$ RunID)

Use-Stores all data for a given run and includes the setup tables which contain details regarding the filter settings and predictions made (table 8). This database is used for all ADAM data reviews.

Table 8. ADAM_Runnn.mdb tables required by ADAM.

[Contains all run data and support data needed to use for data review. ADAM, Automated Data Assurance and Management; $n n$ in file or table name is a unique numeric variable indicating the ADAM Run identifier]

\begin{tabular}{|c|c|}
\hline Table name & Description \\
\hline tbl_ISOutput_Runnn & Output data containing measured and predicted data for this run. \\
\hline tbl_SimSites_Runnn & Sites used to make all predictions for this run. \\
\hline tbl_Prior14Days & $\begin{array}{l}\text { This table is generated by ADAM_REVIEW when the run is opened } \\
\text { for review. }\end{array}$ \\
\hline tbl_FilterDefinitions & \multirow[t]{8}{*}{ Tables are imported from ADAM_SETUP for use by ADAM. } \\
\hline tbl_FilterSettings & \\
\hline tbl_PredEquations & \\
\hline tbl_RunIDs & \\
\hline tbl_PredNotes & \\
\hline tbl_PrelimNotes & \\
\hline tbl_RevNotes & \\
\hline tbl_StationList & \\
\hline
\end{tabular}


Database-ADAM_Query.mdb

Use-Stores tables and queries used for data management during an ADAM run. Data are deleted at run conclusion (table 9).

Table 9. ADAM_Query.mdb tables and queries required by ADAM.

[ADAM, Automated Data Assurance and Management; PCA, principal component analysis]

\begin{tabular}{|c|c|}
\hline Table name & Description \\
\hline tbl_CurrentISOutput & $\begin{array}{l}\text { Stores measured data and ADAM results for current run } \\
\text { only. }\end{array}$ \\
\hline tbl_CurrentTroubleList & Lists all filter trips for current run. \\
\hline tbl_initgagedata & $\begin{array}{c}\text { Loads archival data needed to support filter calculations } \\
\text { so that data analysis can begin with first timestamp. }\end{array}$ \\
\hline tbl_RegressionData & Holds data used in current PCA. \\
\hline tbl_SimSites_Run & $\begin{array}{l}\text { Used by ADAM to generate list of all sites used in } \\
\text { predictions. }\end{array}$ \\
\hline qry_RegressionData_Clean_Hrly_Crosstab1 & Used by ADAM in PCA. \\
\hline qry_RegressionData_Clean_Hrly_Crosstab2 & Used by ADAM in PCA. \\
\hline qry_RegressionData_Clean_Hrly & Used by ADAM in PCA. \\
\hline qry_RegressionData_Clean_Hrly_Group1 & Used by ADAM in PCA. \\
\hline qry_RegressionData_Clean_Hrly_Group2 & Used by ADAM in PCA. \\
\hline qrydate & Used by ADAM in PCA. \\
\hline tbl_VersionHistory & $\begin{array}{l}\text { Lists the history of modifications and (or) updates to } \\
\text { database format. }\end{array}$ \\
\hline \multicolumn{2}{|c|}{ Tables linked to ADAM_Setup.mdb } \\
\hline tbl_StationList & List of stations used by ADAM. \\
\hline
\end{tabular}

\section{Summary}

Data-quality evaluation and estimation of missing data can be a time consuming process, especially for a network as large as Everglades Depth Estimation Network (EDEN). To increase the accuracy of the daily water-level measurements and modeled water surfaces, a Microsoft Excel and Access database tool was created to facilitate quick and accurate quality-assurance review of the real-time data for the EDEN network and to allow estimation or replacement of missing or erroneous data. The ADAM program utilizes inferential sensor technology to effectively and efficiently address data-quality issues by automating many of the processes for quality-assurance checking of data and estimating data when necessary. This user manual describes how to install and operate the ADAM application.

\section{Reference Cited}

Petkewich, M.D., Daamen, R.C., Roehl, E.A., and Conrads, P.A., 2016, Using inferential sensors for quality control of Everglades Depth Estimation Network water-level data: U.S. Geological Survey Scientific Investigations Report 2016-5094, 25 p., http://dx.doi.org/10.3133/sir20165094. 
Manuscript approved July 20, 2016

Publishing support provided by Lafayette Publishing Service Center

For additional information regarding this publication, contact:

Director

USGS South Atlantic Water Science Center

Stephenson Center, Suite 129

720 Gracern Road

Columbia, SC 29210-7651

(803) 750-6100

email: dc_sc@usgs.gov

Or visit the USGS South Atlantic Water

Science Center Web site at:

https://www2.usgs.gov/water/southatlantic/ 
